University of Warwick institutional repository: http://go.warwick.ac.uk/wrap

This paper is made available online in accordance with publisher policies. Please scroll down to view the document itself. Please refer to the repository record for this item and our policy information available from the repository home page for further information.

To see the final version of this paper please visit the publisher's website. Access to the published version may require a subscription.

Author(s): King, P. D. C. and Veal, T. D

Article Title: Conductivity in transparent oxide semiconductors

Year of publication: 2011

Link to published article:

http://dx.doi.org/10.1088/0953-8984/23/33/334214

Publisher statement: None 


\title{
Conductivity in transparent oxide semiconductors
}

\author{
P. D. C. King ${ }^{1}$ and T. D. Veal ${ }^{2}$ \\ ${ }^{1}$ School of Physics and Astronomy, University of St Andrews, North Haugh, St \\ Andrews, KY16 9SS, United Kingdom \\ ${ }^{2}$ Department of Physics, University of Warwick, Coventry, CV4 7AL, United \\ Kingdom \\ E-mail: philip.d.c.king@physics.org
}

\begin{abstract}
Despite an extensive research effort for over 60 years, an understanding of the origins of conductivity in wide band-gap transparent conducting oxide (TCO) semiconductors remains elusive. While TCOs have already found widespread use in device applications requiring a transparent contact, there are currently enormous efforts to (i) increase the conductivity of existing materials, (ii) identify suitable alternatives, and (iii) attempt to gain semiconductor-engineering levels of control over their carrier density, essential for the incorporation of TCOs into a new generation of multifunctional transparent electronic devices. These efforts, however, are dependent on a microscopic identification of the defects and impurities leading to the high unintentional carrier densities present in these materials. Here, we review recent developments towards such an understanding. While oxygen vacancies are commonly assumed to be the source of the conductivity, there is increasing evidence that this is not a sufficient mechanism to explain the total measured carrier concentrations. In fact, many studies suggest that oxygen vacancies are deep, rather than shallow, donors, and their abundance in as-grown material is also debated. We discuss other potential contributions to the conductivity in TCOs, including other native defects, their complexes, and in particular hydrogen impurities. Convincing theoretical and experimental evidence is presented for the donor nature of hydrogen across a range of TCO materials, and while its stability and the presence of interstitial and substitutional species are still somewhat open questions, it is one of the leading contenders for unintentional conductivity in TCOs. We also review recent work indicating that the surfaces of TCOs can support very high carrier densities, opposite to the case of conventional semiconductors. In thin-film materials/devices and, in particular, nanostructures, the surface can have a large impact on the total conductivity in TCOs. We discuss models that attempt to explain both the bulk and surface conductivity based on features of the bulk band structure common across the TCOs, and compare these materials to other semiconductors. Finally, we briefly consider transparency in these materials, and its interplay with conductivity. Understanding this interplay, as well as the microscopic contenders for the conductivity of these materials, will prove essential to the future design and control of TCO semiconductors, and their implementation into novel multifunctional devices.
\end{abstract}

PACS numbers: 72.80.Jc,71.55.-i,73.25.+i,73.61.Le,78.20.-e

Submitted to: J. Phys.: Condens. Matter 


\section{Introduction}

Most wide band-gap materials are electrically insulating, while most conducting materials are opaque to light at visible wavelengths. It is no surprise, therefore, that an exception to this rule - a class of oxide semiconductors which are rather good conductors of electricity while simultaneously being transparent in the visible region of the electromagnetic spectrum - have generated much interest. Despite being known about for well over half a century [1], there remains substantial controversy over the origins of conductivity in these materials, making these one of the least understood classes of semiconductor today.

Termed transparent conducting oxides (TCOs), these materials have already found enormous practical application in devices requiring a transparent contact. Typical examples include: low sheet-resistance current-spreading layers in light-emitting diodes [2,3], to enhance the optical power output; transparent contacts to solar cells [4], to enable current collection with short carrier diffusion lengths, but still allow light to reach the active part of the device; transparent contacts to LCD displays [5]; low thermal emittance (due to the high electrical conductivity) coatings [6], to provide thermal insulation for windows; and electrochromatic, or smart, windows $[7,8]$ whose optical properties can be modulated by charge insertion/extraction through a transparent contact.

The most common TCO used in such applications is Sn-doped $\operatorname{In}_{2} \mathrm{O}_{3}$ (ITO), where carrier densities exceeding $10^{21} \mathrm{~cm}^{-3}$ and resistivities below $10^{-5} \Omega \mathrm{cm}$ have been achieved [7], albeit with substantial variation dependent upon growth technique and conditions. However, indium is a relatively scarce, and consequently expensive, element, which is desired not only for TCOs, but also, for example, as an active component in the rapidly expanding range of InGaN-based optoelectronic, electronic and photovoltaic devices [9-13]. Consequently, much recent work has been focussed on alternative materials [14]. These include the pure and impurity-doped binary materials ZnO, $\mathrm{SnO}_{2}, \mathrm{CdO}$, and $\mathrm{Ga}_{2} \mathrm{O}_{3}$, and also multi-component oxides such as indium-gallium-zincoxide and cubic spinel compounds such as $\mathrm{CdIn}_{2} \mathrm{O}_{4}$ and $\mathrm{SnZn}_{2} \mathrm{O}_{4}$. However, efforts to optimize the properties of TCO materials are frequently hampered by lack of a complete understanding of the origins of their conductivity. Obtaining such understanding is a crucial step towards improvement of current materials and development of a new range of high-performance TCOs for increased functionality and improved device performance. In this review, we concentrate on the $n$-type TCOs - $p$-type TCOs, such as the Cu-based delafossite materials, are considered in passing only in Section 5.

Beyond this, however, TCO materials offer the potential for much more than transparent contacts. It is widely accepted that the staggering advancements seen in the performance of Si-based electronic devices are reaching the limits of what is practically achievable [15], and that a fundamentally new approach is required: oxide materials are tipped to play a leading role in such developments [16-21]. TCOs offer the attractive prospect for the emergence of high-performance transparent electronics, 
offering a wealth of new opportunities in technology and integration [16,17,22], such as completely transparent electronic display screens, or even energy generation from photovoltaic cells invisibly incorporated into the windows of office buildings.

There is reason to be hopeful that the field of TCOs may evolve from its current state of the predominance of transparent contacts to exotic and exciting schemes for transparent electronic devices. Nomura et al. [23] successfully demonstrated the use of $\mathrm{InGaO}_{3}(\mathrm{ZnO})_{5}$ as the channel layer in an all-oxide transparent field-effect transistor (see Fig. 1(a-c)). Crucially, they demonstrated that such a device could exhibit high mobility, stimulating much work on this system $[24,25]$. A second milestone on the road towards oxide electronics can be seen as the observation of the integer [26] and, more recently, fractional [27] quantum Hall effects in $\mathrm{ZnO} / \mathrm{MgZnO}$ heterostructures by Tsukazaki et al. (Fig. 1(d-f)). The realisation of the quantum Hall effect, let alone the fractional quantum Hall effect, is typically restricted to very high-quality semiconductor quantum wells $[28,29]$, and its observation in an oxide material is testament to the staggering advancements in materials synthesis control achieved by Tsukazaki and colleagues. This is further supported by the mobilities achieved in the heterostructures, the best of which was in excess of $180000 \mathrm{~cm}^{2} \mathrm{~V}^{-1} \mathrm{~s}^{-1}$ (Fig. 1(g)), indicating the potential of oxidebased devices for high-mobility applications to rival traditional semiconductors. Such observations are a very encouraging sign towards the development of high-quality oxidebased device architectures [18,30].

We also note here another major discovery suggesting potential for oxide-based materials within electronics: when an interface is formed between the two insulating oxides, $\mathrm{SrTiO}_{3}$ and $\mathrm{LaAlO}_{3}$, a highly conductive interface is obtained [31]. This exhibits a number of very attractive properties including high mobility [31], large magnetoresistance [32], and even superconductivity. [33]. Besides this demonstration of the multifunctional properties obtainable using oxide materials, such observations indicate that interface, or in fact surface, properties can be very different from those of the bulk material, and such effects should not be neglected when considering conductivity in these, and similar, materials.

In any case, to move towards transparent electronic device schemes, we need to understand the reasons why TCOs conduct electricity, so that this can be improved, controlled, and new materials developed with customized properties. There has recently been substantial progress in this area, with conclusions which differ from the conventional perspectives. This paper presents a focussed review of these developments. Initially, native defects are reviewed. Both oxygen vacancies, which are traditionally attributed as the cause of conductivity in TCOs, as well as other isolated defect centres and defect complexes are considered. In Section 3, we consider another candidate, namely hydrogen impurities, that has recently emerged as a leading contender to contribute significantly to conductivity in TCOs. In Section 4, we review recent developments on the surface electronic properties of TCOs, and show that these can also influence the material's total conductivity, in addition to bulk contributions. Overriding tendencies linking the properties of defects, impurities and surface states in these 
materials are discussed in Section 5. Finally, the transparency of TCOs, and in particular its interdependence with conductivity, is discussed in Section 6.

\section{Native defects}

Hampered for many years by difficulty in achieving high quality material, native defects have long been thought to be a dominant factor controlling conductivity in TCOs. In particular, the significant size mismatch between the rather large metal cations and small oxygen anions in these compounds would naïvely be expected to make oxygen vacancies particularly important. In fact, with recent advances in growth techniques, particularly perhaps the advent of novel schemes of molecular beam epitaxy, it has become possible to grow thin-films of oxide materials, as required for device applications, with rather high structural quality [34-40]. Notwithstanding this, however, oxygen vacancies have long been [41], and commonly still are, attributed as the primary cause of conductivity in TCOs. However, this remains a contentious issue, as discussed below.

Much insight has come from theoretical investigations of defect formation energies calculated within the framework of density-functional theory (DFT). It should be remembered, of course, that these calculations are strictly only valid at $0 \mathrm{~K}$. In contrast, growth of oxide materials occurs at elevated temperatures, and is also not an equilibrium process, and so the raw formation energies should always be considered within this context. Furthermore, the well known 'band-gap problem' of DFT [42], coupled with potential errors due to the finite size of supercells used for defect calculations [43, 44], must be corrected in order to understand the formation-energy calculations on an energy scale relevant to experiment. Various schemes have been implemented to account for these issues. Unfortunately, these can lead to rather substantial differences in the calculated formation energies [45] (see, for example, Fig. 2). Notwithstanding these caveats, however, such calculations can still provide much useful information.

\subsection{Oxygen vacancies}

Of all the TCO materials, native defects have been investigated most extensively in $\mathrm{ZnO}$ [46]. For the oxygen vacancy, DFT reveals that the stable charge state moves from the $2+$ donor state to the neutral defect centre as the Fermi level increases (see Fig. 2). However, despite quantitative differences between different calculation schemes, the donor level $([0 / 2+]$ transition level $)$ is generally found to be rather deep, lying well below the conduction band minimum (CBM). This is supported by experiment. By monitoring the optical threshold for ionization of the neutral $\mathrm{V}_{\mathrm{O}}$ centre, detected using electron paramagnetic resonance (EPR), Evans et al. [47] estimated the [0/2+] donor level to lie $\sim 2.1 \mathrm{eV}$ below the CBM. Furthermore, using optical-detected EPR measurements, Vlasenko and Watkins [48] found the [+/0] transition level to lie either $0.9 \mathrm{eV}$ or $2.48 \mathrm{eV}$ above the top of the valence band maximum (VBM). The second of these scenarios is consistent with theoretical calculations [49] indicating a deep donor level. Analysis of 
positron annihilation spectroscopy measurements also found the oxygen vacancy to be a deep donor in $\mathrm{ZnO}$, with an ionization energy of approximately $100 \mathrm{meV}$ [50].

There is also debate over whether oxygen vacancies should be expected, from a theoretical perspective, to occur in significant quantities in as-grown $\mathrm{ZnO}$. The variations in magnitude of the formation energy for the oxygen vacancy between different calculations leads to orders-of-magnitude differences in the concentration of these defects expected from thermodynamic considerations. For example, a high formation energy of the oxygen vacancy for Fermi levels close to the CBM, as calculated by Janotti and Van de Walle [49,51] and Lee et al. [52], would suggest negligible concentrations of such defects under equilibrium conditions. In contrast, from the much lower formation energies calculated by Lany and Zunger [53] and Erhart et al. [54], much higher defect concentrations would be expected, up to $\sim 10^{20} \mathrm{~cm}^{-3}$ under favourable conditions. For a further discussion of these variations between different calculation schemes, see Ref. [45]. The situation is also unclear experimentally. There are several studies which indicate negligible concentrations of oxygen vacancies in as-grown material, with particle irradiation needed to introduce such defects $[48,50]$, while other studies conclude the presence of oxygen vacancies without the need for particle irradiation [55].

Similar properties of the oxygen vacancy have been found in other TCOs. Calculations in $\mathrm{SnO}_{2}, \mathrm{In}_{2} \mathrm{O}_{3}$, and $\mathrm{Ga}_{2} \mathrm{O}_{3}$ again reveal that the oxygen vacancy is a negative-U defect, stable in the $2+$ and neutral charge states $[53,56-59]$. The $[0 / 2+]$ transition levels are again found to be rather deep, although the exact positions are still under debate. As for $\mathrm{ZnO}$, there is significant variation in the magnitude of the calculated formation energies, leading some studies to suggest high concentrations of $\mathrm{V}_{\mathrm{O}}$ under equilibrium conditions [53], while others again find negligible concentrations [57]. Early experimental studies suggested high concentrations of oxygen vacancies at high temperature in polycrystalline $\mathrm{In}_{2} \mathrm{O}_{3}[60,61]$ and $\mathrm{SnO}_{2}$ [62], although this situation may not necessarily hold for high-quality single-crystalline material at room temperature.

There is certainly also experimental evidence that native defects introduced into TCOs can increase the concentration of free carriers in the material. For example, King et al. [63] performed He-ion irradiation studies of $\mathrm{CdO}$, and found an increase and then saturation of the (already high) density of conduction electrons. Speaks et al. [64] reproduced these results for He-ion irradiation, and further showed that irradiation with more massive Ne-ions caused the Fermi level stabilization to occur at higher energies (see Fig. 3). This gives fairly direct proof that native defects can contribute to conductivity in TCOs, although the multiple stabilization levels suggests that this does not occur in a simple manner. While oxygen vacancies are almost certainly created in the irradiation process, the formation of other native defects will likely also occur, and so it is difficult to determine if the observed effect is due to oxygen vacancies, other native defects, complexes, or indeed a combination. Additionally, it should be noted that irradiation of $\mathrm{ZnO}$ with electrons [50] (much lower displacement damage doses), but also oxygen [65] was seen to lead to a increase in resistivity. The detailed situation regarding the electrical behaviour of intentionally-introduced defects across different oxides therefore requires 
further study.

While it is fair to say that this area is still highly controversial, the existing evidence would seem to suggest that the oxygen vacancy does not contribute to conductivity in a simple manner in TCOs. There is a potentially convincing counter-argument: the conductivity in TCOs is often seen to exhibit a pronounced dependence on partial pressure of oxygen during growth $[66,67]$. It would seem logical, therefore, that this conductivity is related to the presence of oxygen vacancies, as has been assumed for many years. However, as will be discussed below, there are several scenarios which can explain this dependence, without requiring isolated oxygen vacancies to be a microscopic source of shallow donors in TCOs.

\subsection{Cation interstitials}

Theoretical calculations have also played an important role in the consideration of other native defects in TCOs. Kılıç and Zunger [56] found that Sn-interstitials were shallow donors in $\mathrm{SnO}_{2}$, and furthermore had a low formation energy, causing them to attribute such defects as the probable source of conductivity in this material. Subsequent theoretical studies [57,68], however, suggested that the formation energy for isolated Sn-intersitials is actually very high for $n$-type material, indicating that such defect centres would be unlikely to be present in significant quantities in $\mathrm{SnO}_{2}$. Furthermore, their calculated migration barrier is very low [57], leading to doubts over the stability of such centres. Interstitial zinc has also been attributed as the cause of $n$-type conductivity in $\mathrm{ZnO}$ [69]. However, as for $\mathrm{SnO}_{2}$, subsequent theoretical studies found rather high formation energies and low migration barriers for the cation interstitial in $\mathrm{ZnO}$, and also other TCO materials $[51,53,70]$. This is also supported experimentally. For example, Zn was shown to diffuse rapidly in $\mathrm{ZnO}$ by secondary ion mass spectrometry [67]. Furthermore, intersitial zinc species were found by ODEPR in $\mathrm{ZnO}$ following electron irradiation, and shown to become mobile well below room temperature [71]. Consequently, cation interstitials are unlikely to be a significant source of room-temperature conductivity in TCOs.

\subsection{Cation vacancies}

From theoretical calculaions, the other isolated native defect that has comparable formation energy to either oxygen vacancies or cation interstitials is the single cation vacancy [51]. In fact, its formation energy decreases with increasing Fermi level, making these especially important for typical Fermi level positions of $n$-type TCOs. However, such defects are acceptors [51,68], and so cannot contribute to this $n$-type conductivity. Rather, these defects may play an important role as compensating centres, limiting the attainable carrier densities and conductivities of TCOs. Indeed, this defect been identified as the dominant compensating acceptor in both as-grown and electronirradiated $n$-type $\mathrm{ZnO}$ from positron annihilation spectroscopy measurements [50,72]. 


\subsection{Defect complexes}

Much attention has also been paid to defect complexes, which can substantially change the formation energetics relative to those of isolated defect centres. For example, Kim and Park [73] argued that attractive interactions between the oxygen vacancy and zinc interstitial in $\mathrm{ZnO}$ lowered their formation energy and made them significantly more likely to contribute to $n$-type conductivity. Tang et al. [74] found a similar effect in $\mathrm{In}_{2} \mathrm{O}_{3}$. Look et al. [75] have argued that a complex of the Zn-interstitial with a nitrogen impurity on the oxygen site is also a strong candidate for a shallow donor in ZnO. In $\mathrm{SnO}_{2}$, Godinho et al. [68] found that oxygen vacancies compensated through tin reduction could be present in significant quantities, and contribute to the $n$-type conductivity. More generally, they concluded that isolated defects often relax to give completely different defect structures, a feature which is neglected in the majority of calculations of defect formation energies. Combined with difficulties in unambiguous identification of defect complexes in experiment, it it is clear that much work remains in order to obtain a detailed picture of defects and their complexes in TCOs, and their possible role in conductivity.

\subsection{Persistent photoconductivity}

Although as discussed above, it seems likely that oxygen vacancies are typically rather deep donors in TCOs, it could still be possible for them to contribute towards conductivity in these materials. In both $\mathrm{ZnO}[53,76]$ and $\mathrm{In}_{2} \mathrm{O}_{3}$ [53], Zunger and colleagues have proposed that the oxygen vacancy leads to the population of the conduction band with free carriers via a persistent photoconductivity (PPC) mechanism. In this model, upon photoexcitation, two electrons are promoted from the deep non-conductive $V_{\mathrm{O}}^{0}$ level to a metastable conductive shallow state [77]. An energy barrier prevents the transition back to the non-conducting ground state, allowing such photoconductivity to persist, providing a model for the conductivity of TCOs dependent on oxygen vacancies. There is debate, however, whether the energy barrier to prevent such a transition back to the ground state is sufficiently large to achieve PPC in practice. For example, in ZnO, Janotti and Van de Walle [51] find that this barrier would be sufficient to allow PPC at low temperature, but not at room temperature.

It is apparent that the situation involving native defects and their contribution to conductivity in TCOs is not entirely clear. There is both experimental and theoretical evidence that they are important in certain situations, but they seem unlikely to be the sole mechanism responsible for conductivity in these materials. The common assignment of oxygen vacancies as the dominant donor defect in TCOs seems to be, at best, an over simplification. In addition to other native defects, there are several distinct candidates that appear to have an important role in conductivity, as will be discussed in the following sections. 


\section{Hydrogen in oxides}

\subsection{Donor nature of hydrogen}

Hydrogen is well known to be an important impurity in semiconductors [78]. It is hard to remove from growth environments, and so may be expected to be present as an impurity in the majority of materials, where it is normally electrically active. Interstitial hydrogen is almost universally accepted to be a negative- $U$ defect, stable in the $1+$ and 1 - charge states but not in the neutral state [79]. Hydrogen, therefore, forms an acceptor (donor) state when the Fermi level lies above (below) the so-called $\mathrm{H}[+/-]$ transition level, which lies mid-way between the acceptor $(\mathrm{H}[0 /-])$ and donor $(\mathrm{H}[+/ 0])$ levels. In a conventional semiconductor such as GaAs, this transition level lies approximately in the middle of the band gap [80]. Consequently, for Fermi levels close to the valence band ( $p$-type conductivity), hydrogen will act as a compensating donor, whereas for Fermi levels approaching the conduction band ( $n$-type conductivity), hydrogen will act as a compensating acceptor. Thus, in conventional semiconductors, hydrogen always counteracts the prevailing conductivity.

This picture, however, seems inconsistent with work on oxides from as early as the 1950s, where hydrogen diffusion into $\mathrm{ZnO}$ was found to increase its conductivity [81]. From DFT calculations, Van de Walle and colleagues [79,82,83] showed that interstitial hydrogen can indeed act as a donor even in $n$-type $\mathrm{ZnO}$, finding that the $\mathrm{H}^{+}$charge state is stable for all Fermi level positions within the band gap (see Fig. 4(b)). This finding means that for $n$-type material, with the Fermi level close to, or even somewhat above, the CBM, the donor-state is still favourable. Consequently, hydrogen could be a cause of conductivity in $\mathrm{ZnO}$, rather than playing its usual role as a compensating defect centre, explaining the measured increase in conductivity observed upon hydrogen diffusion into $\mathrm{ZnO}$.

Cox et al. [84] performed muon spin rotation and relaxation spectroscopy $(\mu \mathrm{SR})$ measurements on $\mathrm{ZnO}$, investigating muonium $\left(\mathrm{Mu}=\left[\mu^{+}, e^{-}\right]\right)$as a lightisotope analogue of hydrogen. While hydrogen can be difficult to directly assess spectroscopically, the well-defined creation and decay properties of muons has led to significant success in the use of $\mu \mathrm{SR}$ as a spectroscopic probe of isolated defect centres in semiconductors that differ from hydrogen only by a mass factor $\left(m_{\mathrm{Mu}} / m_{\mathrm{H}} \approx 1 / 9\right)$ and the associated small difference in zero-point energy [85]. While $\mu \mathrm{SR}$ studies of conventional semiconductors such as Si [86] or GaAs [87] found charge transition levels for muonium lying deep within the band gap, as would be expected for the conventional behaviour of hydrogen in these materials, such measurements [84] provided the first spectroscopic evidence of the donor-nature of hydrogen in $\mathrm{ZnO}$, summarized

in Fig. 5. At low temperatures, a paramagnetic $\mathrm{Mu}^{0}$ signal is seen to co-exist with the diamagnetic $\mathrm{Mu}^{+}$signal (Fig.5(a)), indicating the presence of neutral muonium. The ionization behaviour of this paramagnetic component with increasing temperature, coupled with the small effective hyperfine splitting (evident from the small splitting of the paramagnetic satellite lines in the frequency domain, Fig. 5(b)) gives strong evidence 
for a shallow donor state of muonium. By analogy, a shallow donor state of hydrogen would also be expected, which was soon-after confirmed by EPR measurements [88]. These works stimulated a flurry of studies on the presence and local bonding environment of interstitial hydrogen in $\mathrm{ZnO}[46,89-98]$. In addition, hydrogen has been shown as a viable intentional $n$-type dopant [99] and co-dopant with $\mathrm{Al}$ [100] and Ga [101] in $\mathrm{ZnO}$ grown by a variety of techniques ranging from sputtering methods [99] to chemical solution deposition [100]. Free electron densities as high as $6 \times 10^{20} \mathrm{~cm}^{-3}$ have been achieved by doping with hydrogen along [99]. This collection of theoretical and experimental studies potentially provides a new paradigm for the origins of conductivity in TCOs: hydrogen, a ubiquitous impurity which is likely to be present in virtually all as-grown material, may be the unintentional dominant donor. However, it is important to consider if (i) this is specific to $\mathrm{ZnO}$, or is general across the TCOs, (ii) whether it is stable, particularly at elevated temperatures, and (iii) whether the concentrations of this impurity species are likely to be high enough to account for the measured conductivities of TCOs. These issues are addressed in the following sections.

\subsection{Universality across TCOs}

Several subsequent $\mu \mathrm{SR}$ studies have revealed the shallow donor-nature of muonium in the TCO materials $\mathrm{In}_{2} \mathrm{O}_{3}$ and $\mathrm{SnO}_{2}$ [102], $\mathrm{CdO}$ [103], and $\mathrm{Ga}_{2} \mathrm{O}_{3}$ [104] (see, for example, Fig. 6). In all cases, a paramagnetic component is observed at low temperatures, which is quenched with increasing temperature. The variations in amplitudes are well described by ionization models with small activation energies indicative of a shallow donor state. The effective hyperfine splitting of the paramagnetic components are very small (see the inset to Fig. 6(c)), indicating an extended wavefunction. The shallow-donor radius and binding energy determined from the $\mu \mathrm{SR}$ studies are in good agreement with simple estimates within a hydrogenic model $[102,104]$. This gives a strong spectroscopic indication that the shallow-donor state of hydrogen in conducting oxides is not limited to $\mathrm{ZnO}$, but rather is general across the TCOs. Further experimental studies have also observed or inferred the donor nature of hydrogen in TCOs. For example, an increase in measured carrier concentration and additional Moss-Burstein shift of the optical absorption edge was observed when hydrogen is diffused into $\mathrm{CdO}[63,105]$. Indeed, $\mathrm{CdO}$ films grown by metal-organic chemical vapour deposition, where hydrogen is present in large quantities in the precursor gas, have high initial carrier densities, which reduce upon annealing in ultra-high vacuum. This is consistent with a dissociation of hydrogen, leading to a corresponding reduction in carrier density, with annealing [63]. Annealing nominally undoped $\mathrm{SnO}_{2}$ in a hydrogen atmospheres was shown to lead to an increase in the conductivity, concurrent with the appearance of an $\mathrm{O}-\mathrm{H}$ stretch mode in infrared absorption measurements, confirming the donor nature of interstitial hydrogen in $\mathrm{SnO}_{2}$ [106]. Meanwhile, hydrogen-doping during growth has been found as an effective method to produce highly conducting $\mathrm{In}_{2} \mathrm{O}_{3}$ films [107]. Indeed, hydrogendoped $\mathrm{In}_{2} \mathrm{O}_{3}$ has been suggested as a suitable contact for use in Si-based heterojunction 
solar cells [108].

Such experimental observations are also supported by theoretical calculations. Kılıç and Zunger [109] and Xiong et al. [110] found that hydrogen forms a shallow-donor state in $\mathrm{CdO}$ and $\mathrm{SnO}_{2}$, in addition to $\mathrm{ZnO}$. Singh et al. [57] also found the shallow donor state to be stable for interstitial hydrogen in $\mathrm{SnO}_{2}$, while Limpijumnong et al. [58] and Varley et al. [59] found the same to hold for $\mathrm{In}_{2} \mathrm{O}_{3}$ and $\mathrm{Ga}_{2} \mathrm{O}_{3}$, respectively. These theoretical studies support the conclusion from the experimental results that interstitial hydrogen forms as a shallow donor, even in $n$-type material, across the class of TCOs. The overriding reasons for this are discussed further in Section 5.

\subsection{Stability}

The above discussions concentrate on isolated interstitial hydrogen. While these studies show it to be a shallow donor in TCOs, it is not a priori clear that such species will be stable at elevated temperatures, for example during growth or even some device operating temperatures, where the conductivity of TCOs is known to persist. Indeed, deuterium was observed to diffuse easily in $\mathrm{ZnO}$ with an activation energy of only $170 \mathrm{meV}$ [111]. While diffusion of hydrogen into $\mathrm{ZnO}$ was seen to lead to an increase both in electron concentration and an associated local vibrational $\mathrm{O}-\mathrm{H}$ stretching mode observed in infrared spectroscopy [90], both were subsequently substantially reduced by annealing at only $150^{\circ} \mathrm{C}$ for 30 mins [96]. Indeed, Jokela and McCluskey [95] showed that the O-H IR absorbance peak and carrier density of hydrogenated $\mathrm{ZnO}$ both decreased with the same functional form even at room temperature, as shown in Fig. 7, which they explained via formation of neutral $\mathrm{H}_{2}$ molecules. A similar decrease in the $\mathrm{O}-\mathrm{H}$ stretch mode with room-temperature annealing was also observed in $\mathrm{SnO}_{2}$ [106].

While the correlation of such spectroscopic and electrical measurements provide good evidence that the interstitial state of hydrogen is a donor in $\mathrm{ZnO}$, and can contribute carriers directly into the conduction band, it also reveals that this species is unlikely to be sufficiently stable to account for the residual background concentration of carriers in TCOs at room temperature, let alone their persistence at elevated temperatures. Wardle et al. [112] investigated the diffusion of hydrogen in ZnO from DFT calculations, finding low migration barriers, suggesting that interstitial hydrogen is indeed very mobile in $\mathrm{ZnO}$. However, they suggested that hydrogen could still contribute to conductivity in $\mathrm{ZnO}$, even at elevated temperatures, if it becomes trapped at other existing defect centres. This is consistent with spectroscopic observations of multiple H-related donors in this material [93, 94].

Janotti and Van de Walle [83] showed that substitutional hydrogen on the oxygen

site in $\mathrm{ZnO}$ also forms a shallow donor, in a so-called multicentre bond configuration (see Fig. 4). While the formation energy of this species is very similar to that of isolated interstitial hydrogen (Fig. 4(b)), it is significantly more thermally stable. Indeed, the calculated energy required for the conversion $\mathrm{H}_{\mathrm{O}} \rightarrow \mathrm{H}_{i}+V_{\mathrm{O}}$ was calculated to be $3.8 \mathrm{eV}$. Furthermore, substituting for oxygen causes the formation energy of this configuration 
of hydrogen to be intimately linked with the oxygen chemical potential, reconciling the explanation of hydrogen as a source of conductivity in TCOs with the known dependence of conductivity on oxygen partial pressure during growth. Signatures of such a multicentre bond configuration have recently been identified in a combined Raman scattering, IR absorption, photoluminescence, and photoconductivity study by Lavrov et al. [97].

Subsequent calculations have revealed that the shallow donor state of substitutional hydrogen is also stable, and has similar formation energies to the interstitial state, in other TCOs including $\mathrm{SnO}_{2}$ [57] (4-centre bond) and $\mathrm{In}_{2} \mathrm{O}_{3}$ [58] (5-centre bond).

Experimental studies are somewhat less advanced, however, in these other TCOs as compared to ZnO. While initial experiments on interstitial hydrogen, discussed above, suggest that the situation in these other TCO materials is similar to that in $\mathrm{ZnO}$, further experiments should be performed to confirm this, in particular with regard to the multi-centre bond substitutional species.

\subsection{Origin of the bulk n-type conductivity?}

From the extensive theoretical and experimental studies discussed above, there seems little doubt that hydrogen has a strong propensity to form as a shallow donor in TCOs, and consequently must be considered in any picture of conductivity in these materials. Substitutional hydrogen, with its greater thermal stability than interstitial hydrogen and the naturally occurring mechanism for a dependence of conductivity on oxygen partial pressure during growth, is certainly an attractive candidate for the unintentional dominant donor leading to conductivity in TCOs. However, the situation in real materials is unlikely to be this simple. As discussed above, multiple species of hydrogenrelated defects are known to exist, and interstitial hydrogen, potentially trapped at other native defect centres, may also contribute to the material's conductivity. The role of native defects, whether in complexes with hydrogen or as a contribution to conductivity on their own, can also not be ruled out, and in reality, most materials likely contain contributions to their total conductivity from a variety of microscopic sources.

It is also worth noting that any theory of conductivity based predominantly around impurities such as hydrogen can only be valid where such impurities are present in significant quantities in the as-grown material. While hydrogen is hard to remove from most growth environments, it is still difficult to assess what the concentration of this impurity really is. Hydrogen has also been proposed as a source of conductivity in another novel semiconductor material InN, which is actually similar in many respects to TCOs (see Section 5). In that material, there is currently intense debate over whether hydrogen can be seen as the dominant source of conductivity, whether it is present in sufficient quantities, and whether it remains dominant over native defects over the whole Fermi level range spanned in existing material [113-122]. Such considerations may be expected to apply to TCO materials as well, and further investigations in this area are still required. Material grown using different techniques may fall into regimes 
where different impurities or native defects dominate the conductivity. For example, the situation may be expected to differ between ultra-high vacuum based techniques such as molecular-beam epitaxy and solution-based methods of growth. In addition, as will be discussed in the next section, it is not sufficient to consider only the bulk of these materials, as the surface, and very possibly the interface, can have pronounced effects on the total conductivity. What may be said with some certainty, however, is that hydrogen incorporation should be limited as far as possible if trying to grow highquality semiconducting material for use in novel electronic devices, whereas deliberate doping of TCOs by hydrogen may offer a suitable route to obtaining highly conducting TCO films for use as transparent contacts.

\section{Surface conductivity}

Just as at a defect or localized impurity centre, the breaking of translational symmetry at the surface of a material allows electronic states to exist which differ from those in the bulk [123-126]. Such surface states have either a donor- or an acceptor-like character, and so are charged dependent on their occupancy - unoccupied donor surface states are positively charged, while occupied acceptor surface states are negatively charged. To reduce energy, the carriers in the near-surface region of the material can rearrange to screen the surface charge. This leads to macroscopic space-charge regions where the carrier concentration varies markedly in the near-surface region of a material. This is important in device applications, where an electrical contact must always be made to the surface of a material. However, in thin-films of materials, and in particular in nanostructures, where the surface to bulk ratio can become large, redistribution of charge at the surface of a material can have a pronounced effect on the total conductivity. Consequently, we discuss this here in relation to conductivity in TCOs.

At the surface of all ( $n$-type $\ddagger$ ) conventional semiconductors, for example in Si $[127,128]$ and GaAs $[129,130]$, negatively-charged acceptor-like surface states cause a pinning of the Fermi level close to the centre of the fundamental band gap. Consequently, the Fermi level at the surface lies lower relative to the band edges than in the bulk of the semiconductor - the bands are said to bend upwards relative to the Fermi level. This leads to a region depleted of carriers near to the surface. It is the net positive charge of this depletion region that balances the negative surface charge, maintaining charge neutrality. This was long thought to be the situation at the surface of TCOs [131-133]. However, recent work performed on high-quality, relatively low carrier density, singlecrystalline material has rather suggested the opposite situation is rather the intrinsic one.

Investigating the (100) surface of nearly non-degenerate bixbyite $\mathrm{In}_{2} \mathrm{O}_{3}$ films grown by MBE, King et al. $[134,135]$ used photoemission spectroscopy to show that the Fermi

$\ddagger$ For $p$-type conventional semiconductors, opposite considerations apply, with a positive surface charge from unoccupied donor-like surface states leading to downward band bending. This again leads to a depletion of the majority carriers at the surface. 
level at the surface is pinned $3.4 \mathrm{eV}$ above the VBM (see Fig. 8(a)). In contrast, from IR reflectivity (Fig. 8(b)) and Hall effect measurements, the bulk Fermi level was found to lie close to the $\mathrm{CBM}(\sim 2.95 \mathrm{eV}$ above the VBM). This indicates that the Fermi level is pinned substantially above its bulk value at the surface, indicating a strong downward band bending (Fig. 8(c)), and consequently a pronounced increase in electron density approaching the surface of the material (Fig. 8(d)). Such a surface electron accumulation was found to be remarkably independent of surface orientation or even bulk polymorph, with a very similar pinning level of the surface Fermi level, and resulting degree of electron accumulation, at the (001) and (111) surface of bixbyite $\operatorname{In}_{2} \mathrm{O}_{3}$, and the (0001) surface of rhombohedral $\mathrm{In}_{2} \mathrm{O}_{3}$ [135]. In the photoemission spectra, finite spectral intensity can be observed close to the Fermi level in $\mathrm{In}_{2} \mathrm{O}_{3}$ (see the magnified spectrum in Fig. 8(a)). While this has previously been attributed to photoemission from occupied bulk conduction-band states, the data shown in Fig. 8 is from a sample which is virtually non-degenerate in the bulk. This indicates that such a peak actually results from carriers within the surface electron accumulation layer, rather than from the bulk. Zhang et al. [136] employed energy-dependent photoemission, utilizing the inherent variation in surface specificity with photon energy to support this conclusion, providing spectroscopic confirmation of the increased electron density at $\operatorname{In}_{2} \mathrm{O}_{3}$ surfaces. Via extrinsic Sn-doping to increase the bulk carrier concentration, it is possible to quench the electron accumulation $[134,135]$. This provides a mechanism to reconcile these recent results with earlier studies which suggested a depletion of electrons at the surface [131-133].

A similar electron accumulation has been observed at the surface of $\mathrm{CdO}$ [63], while in $\mathrm{ZnO}$ this phenomenon has long been known at the surface of hydrogenated material [137]. Its existence at the surfaces of $\mathrm{ZnO}$ that has not been deliberately hydrogenated was still attributed to residual hydrogen contamination [138]. Allen et al. [139] and Schmidt et al. [140] have recently argued that electron accumulation is actually intrinsic to $\mathrm{ZnO}$, as in the other TCOs.

The surface of $\mathrm{SnO}_{2}$ can exhibit surface electron depletion or accumulation, depending on the stoichiometry of the surface and the bulk Fermi level. Similarly to $\mathrm{ZnO}$, absorption of anion species leads to electron depletion, while cation absorption leads to accumulation, making $\mathrm{SnO}_{2}$ a good material for gas sensing via corresponding changes in conductivity. The presence of an electron accumulation layer at the surface of $\mathrm{SnO}_{2}$ under certain conditions has long been known [141], and recent work suggests that it may after all be the 'intrinsic' state' of the surface [142] as for the materials discussed above. While no direct evidence of electron accumulation has been reported for $\mathrm{Ga}_{2} \mathrm{O}_{3}$, thin films of this material have been successfully used as gas sensors with sensitivity to chemisorption of donor-like species such as $\mathrm{H}_{2}$ and $\mathrm{CO}$ [143]. This behaviour was explained in terms of an electron accumulation layer model of the $\mathrm{Ga}_{2} \mathrm{O}_{3}$ surface [144]. The presence of an electron accumulation is also suggested by recent photoemission data from $\mathrm{Ga}_{2} \mathrm{O}_{3}$, where the surface Fermi level can be seen to be approximately $4.5 \mathrm{eV}$ above valence band maximum, close to the conduction band minimum [145]. 
Surface electron accumulation layers are known to have a pronounced effect on the measured conductivity and its temperature dependence [139,140,146,147], and so such a surface contribution cannot be ignored when considering conductivity in TCOs. Recent measurements also indicate that an accumulation of electrons can also be induced at the surfaces of other, more complex, oxides such as $\mathrm{SrTiO}_{3}$, indicating that this phenomenon may indeed be rather general across the oxide semiconductors [148, 149].

The strong downward band bending associated with the electron accumulation creates a confining potential well at the surface of the material. This causes the conduction band states to become quantized into two-dimensional subbands (the electron accumulation can be seen as a two-dimensional electron gas (2DEG)). This two-dimensional electronic structure at the surface of $\mathrm{CdO}$ has been observed directly by angle-resolved photoemission spectroscopy [150,151], as shown in Fig. 9.

Such measurements [150] have recently revealed an unexpectedly large importance of many-body interactions within the electron accumulation layer, which leads to a depth-dependent shrinkage of the semiconductor band gap. Consequently, the band gap at the interface of TCOs with other materials may also be modified from its bulk value, and this should be investigated for its potential influence on the properties of these electrical contacts.

Aside from surface conductivity of TCOs, one should also consider a possible conductivity at the interface with the substrate or with other oxide layers. There are both intrinsic and extrinsic (defect-related) potential contributions to interface conductivity. As discussed in the introduction, the interface between $\mathrm{ZnO}$ and $\mathrm{MgZnO}$ has been shown to support a high-mobility 2DEG of sufficient quality to exhibit the integer or even fractional quantum Hall effect [26, 27], while the interface between two insulating oxides $\mathrm{SrTiO}_{3}$ and $\mathrm{LaAlO}_{3}$ can support a high-mobility 2DEG [31] wth a number of novel features $[32,33]$. Clearly, oxide interfaces offer potential for the realisation of a variety of novel physical phenomena and conductivities that differ drastically to those in the bulk of the material. In addition to these "designer" interface 2DEGs, we note here that, in the similar material $\mathrm{InN}$, dislocations that are generated at the InN/substrate (or buffer layer) interface have been reported to be a source of $n$-type conductivity, causing an unintentional increase in electron density approaching the interface $[120,152-154]$. Given the propensity for donor-like defects and impurities in TCOs, it seems plausible that a similar effect could occur at a latticemismatched TCO/substrate interface. A preliminary investigation [155] of the filmthickness dependent electronic properties of $\mathrm{In}_{2} \mathrm{O}_{3}$ suggests a similar phenomenon may indeed occur here. However, detailed investigations of other materials, substrates, and effects of lattice mismatch, strain, and the precise mobility variations are required to understand the full nature of the interfaces of this and other TCOs. 


\section{Overriding explanation?}

Given the tendency for impurities such as hydrogen, several native defects, and also surface states to all be donors in TCOs, as discussed in the above sections, it may be questioned what drives this propensity towards $n$-type, rather than simply compensating or $p$-type states. King et al. $[63,134]$ have argued that this can be understood within the concept of the charge neutrality level (CNL) [156,157]. Such localised impurities, defects, and surfaces are all examples of broken symmetries - that is, the perfect translational symmetry of the lattice is lost. Within the CNL model, the charge state of the defect centre is determined by the donor or acceptor character of the associated virtual gap state (ViGS). Such ViGS result from evanescent states, associated with the complex wavevectors allowed in the solution of Schrödinger's equation in the absence of the full lattice periodicity, subject to the matching of their wavefunction tails with bulk states $[158,159]$. As these states derive from the bulk electronic bands, they have predominantly donor (acceptor) character closer to the valence (conduction) band. The branch point of these states, where they have equal donor- and acceptor-like character, represents the CNL of the semiconductor. Consequently, there is a tendency for native defects, hyrdogen, and surface states to all be donor-like if the Fermi level lies below the CNL, and acceptor-like if the Fermi level is above the CNL (see Fig. 10). While it should be noted that this is not a microscopic model, it does explain the overriding tendencies for the electronic properties of materials, and incorporates several widelyobserved phenomena including the effects of particle irradiation on electronic properties (the amphoteric defect model) [160-162], the reported universality of the $\mathrm{H}[+/-]$ charge transition level of hydrogen between materials [79], and the observations of the similar electrical characteristics of native defects, hydrogen impurities and surface states in semiconductors [63], as discussed above for TCOs.

Taken together, the above considerations strongly suggest that the CNL lies above the CBM in TCOs, so that donor-ViGS remain favourable even for Fermi levels into the conduction band. Indeed, from direct measurements, measurements of valence band offsets (allowing band alignment relative to the CNL), and theoretical calculations [63,135,163-167], the CNL has been found to occur above the CBM across the TCOs, as summarized in Fig. 11. This provides an overriding explanation of the donor-like nature of defects, impurities, and surfaces in TCOs, and hence the origins of their conductivity.

An obvious question, therefore, is what causes the CNL to lie above the CBM in TCOs. Indeed, as the CNL lies at the branch point of gap states, it seems intuitive that this should be within the fundamental semiconductor band gap, and this is indeed the case in conventional semiconductors such as $\mathrm{Si}$ and GaAs (see Fig. 11). However, the gap states result from localized (in real space) defect centres. Thus, they are extended in $\mathbf{k}$-space, and so their character derives from the complex band structure across the Brillouin zone, rather than just at the $\Gamma$-point. The large size mismatch between the cation(s) of a TCO and the oxygen anion, and the high electronegativity (and therefore 
low O $2 s$ orbital energy) of the anion, cause the electronic band structure of TCOs to exhibit a single low-lying CBM at $\Gamma$. This is shown for $\mathrm{In}_{2} \mathrm{O}_{3}$ in Fig. 12 [170], but can also be seen in calculations of the electronic structure of the other TCOs [68,171-174]. Such a band structure means that the Brillouin-zone-averaged band gap, and consequently also the CNL [175], are situated above the low-lying CBM, rather than within the fundamental band gap $[63,167]$. We also note that the highly dispersive conduction band, and so relatively small effective mass, is crucial in obtaining sufficiently high mobilities in these materials to ensure adequate conductivity.

This model provides a simple explanation for the propensity towards high $n$-type conductivity of TCOs, in contrast to conventional semiconductors such as Si and GaAs. As can be seen from Fig. 11, it also suggests that TCOs and the semiconductor InN should exhibit many similar properties, and this is supported by experiment $[12,120,169]$. In both cases, the CNL lying above the CBM can be seen as a result of the large size and electronegativity mismatch between the cation and anion [169]. In contrast, in materials where the CNL lies close to the VBM, such as in GaSb (Fig. 11), the opposite properties to those considered here would be expected, explaining GaSb's propensity for unintentional $p$-type conductivity, surface hole accumulation [176], and the acceptor nature of hydrogen [177]. Consequently, to obtain a $p$-type TCO, the CNL must be located much lower within the fundamental band gap, as in $\mathrm{CuAlO}_{2}$ (Fig. 11) [166], where p-type conduction has been realized [178]. Consequently, the CNL model provides a useful starting point for the understanding and design of TCO materials (of both polarities). It does not, however, elucidate the exact microscopic origins of the conductivity, or distinguish between different candidates. As outlined in the preceding sections of this review, much work still remains to answer these questions with certainty. We also note that this model may not be directly valid in all cases. For example, an alternative scheme has been suggested in unconventional TCOs such as $\mathrm{Ca}_{12} \mathrm{Al}_{14} \mathrm{O}_{33}$, where a defect band crossing the Fermi level was suggested to be the source of conductivity in this very wide band gap material [179]. A recent first-principles investigation by Medvedeva and Hettiarachchi [180] into single- and multi-component TCOs has also identified a number of microscopic properties, such as the overlap of metal $s$-states with oxygen $p$-states, oxygen coordination, and the doping with transition metal elements, which could be important to effectively obtain high conductivity material while maintaining optical transparency.

\section{Transparency}

Of course, conductivity is only half of what is required for a TCO - transparency at visible frequencies is equally important. Furthermore, the transparency is not independent of the conductivity, but rather intricately linked to it. Consequently, in this section we briefly review the different contributions to optical absorption that must be considered in these materials, their relation to the conductivity, and outline some particular features of optical transitions in selected materials. 
The most obvious effect limiting transparency is direct interband optical absorption between the top valence band and the bottom conduction band. As the carrier concentration increases, the Fermi level in these degenerate materials shifts to higher energies above the CBM. Consequently, the onset of optical absorption due to such interband transitions shifts to higher energies with increasing doping [181,182], as shown in Fig. 13. In addition to this Moss-Burstein shift, which widens the optical energy gap, it has been found that many-body interactions lead to a shrinkage of the fundamental band gap with increasing free-carrier concentration [183]. This has been employed in the analysis of optical absorption in many TCOs [184-189], and has recently been shown to have a pronounced depth-dependent effect on the band gap size within their surface electron accumulation layers [150], as mentioned above. However, the origins of such band gap narrowing have also recently been questioned [190], with hybridization between states from the dopant impurity atoms and the host conduction band states rather than many-body interactions between the free carriers attributed as the cause of the bandgap shrinkage. While the details of these effects require further investigation, it does not change the qualitative trend of the increased Moss-Burstein shift with higher carrier densities in these materials.

A number of distinct optical transitions are also important. Once the conduction band becomes populated with carriers (that is, the material becomes degenerately doped), transitions between occupied states of the bottom conduction band and unoccupied states in higher-lying conduction bands become possible. As shown in Fig. 13, with increasing carrier concentration, and therefore increasing Fermi level position above the CBM, the energy for direct transitions between the lower and higher conduction bands reduces. This has recently been suggested as a potentially important contribution to the total optical absorption, for example, in $\mathrm{In}_{2} \mathrm{O}_{3}$ [170]. Furthermore, for a degerately-doped semiconductor, a free-carrier absorption process can occur, where a carrier from below the Fermi level is excited to an empty state above the Fermi level. The energy scale associated with such absorption is often in the infrared region of the spectrum, but this can increase towards the visible as the free-carrier concentration, and hence the plasma frequency, increases. Consequently, maintaining transparency in TCOs is a competition between increasing the energy of fundamental valence-to-conduction band transitions (requiring large fundamental band gaps, but also assisted by high carrier-densities, and so a large Moss-Burstein shift), with limiting the contribution of inter-conduction-band transitions which could lower the fundamental absorption edge (requiring lower carrier concentrations), and limiting the optical absorption at the infrared end of the spectrum due to free-carrier absorption (again requiring lower carrier concentrations). This must all be balanced with the requirements for relatively high carrier concentrations, and also high mobilities, to maintain good conductivity.

There are additional features which can help transparency to be maintained in several TCO materials. For example, in $\mathrm{CdO}$, the fundamental band gap is only $\sim 1 \mathrm{eV}$. However, this band gap is indirect [191]. Consequently, only phonon-assisted optical transitions are allowed from the VBM to the CBM, with small associated 
transition matrix elements, and significant optical absorption due to direct interband transitions does not occur until some $1.2 \mathrm{eV}$ above the fundamental band gap [189]. This enables doped $\mathrm{CdO}$ to be rather transparent at visible frequencies, even though the fundamental band gap would seem to be too low to allow this. In some other TCOs such as $\mathrm{In}_{2} \mathrm{O}_{3}[135,170,192], \mathrm{SnO}_{2}$ [193], $\mathrm{SnZn}_{2} \mathrm{O}_{4}, \mathrm{SnCd}_{2} \mathrm{O}_{4}$, and $\mathrm{CdIn}_{2} \mathrm{O}_{4}$ [194] symmetry dictates that interband transitions between the top valence bands and the bottom conduction band are either dipole forbidden or have only minimal dipole matrix elements, as represented for $\mathrm{In}_{2} \mathrm{O}_{3}$ in Fig. 14(a). This therefore extends the range of transparency in these materials over what would be expected considering the fundamental band gap alone. This presents novel opportunities for tuning the band edge absorption coefficient by alloying materials where the lowest-energy transitions are either dipole allowed or forbidden, as for example in $\mathrm{ZnO}$ and $\mathrm{In}_{2} \mathrm{O}_{3}$, respectively, as shown in Fig. 14(b) [195]. A detailed discussion of multi-component TCOs is beyond the scope of this review, and the interested reader should consult the review by Walsh et al. [196].

\section{Conclusions and Outlook}

The above discussions have illustrated a number of potential sources of conductivity in transparent conducting oxides. It seems apparent that conductivity in these materials is not simply due to oxygen vacancies, as has often been assumed in the past. While these defects may play some role, an influence of other native defects, defect complexes and impurities is likely to be important. In particular, perhaps, the hydrogen impurity appears to be a strong contender for an important microscopic donor in TCOs. There is convincing theoretical and experimental evidence that interstitial hydrogen is a donor across the $n$-type TCOs, in contrast to its behaviour in other semiconductors. While alone it may not be stable at growth and device-operating temperatures, substitutional donor hydrogen (essentially a hydrogen impurity localized in an oxygen vacancy) and trapping of interstitial hydrogen at native defects both seem suitable possibilities to obtain stable donor hydrogen centres. This serves to illustrate the richness of defect physics in these materials, and the importance of the interplay between different defects. In any real material, there will likely be a combination of defects/impurities contributing to conductivity, and so it may not be appropriate to define any single dominant donor. Further, the importance of a particular defect centre may vary dependent on, for example, growth technique or conditions.

Unlike conventional semiconductors, which exhibit a depletion of majority carriers at the surface, TCOs have been shown to support a build-up of electronic charge there. This can have a pronounced influence on the total conductivity in thin-film and nanoscale materials, as well as being important for electrical contacts in devices, and so the surface properties of these materials should not be neglected. A general understanding of the strong propensity driving the donor nature of not only bulk defects and impurities, but also these surface states, comes from universal properties of the bulk 
band structure of TCOs; the characteristic single low-lying conduction band minimum lies below the so-called charge neutrality level. While not providing a microscopic explanation of conductivity, it does provide a useful tool to consider TCOs within the context of other semiconductors. It is also apparent that maintaining the desired transparency of these materials requires a delicate interplay of the influence of free carriers on valence to conduction interband, conduction to higher conduction interband, and intra-conduction band (free-carrier) optical absorption processes, with transition matrix elements also play a crucial role.

We note here that TCOs are not the only way to make a transparent contact to a material. Conducting polymers [197-199] can exhibit rather high optical transparency [200]. Meanwhile, thin-films of carbon nanotubes have been created which are both conducting and highly transparent [201,202]. Such alternatives to TCOs may find application in particular within flexible electronic devices. However, it seems likely that TCOs will remain a cornerstone of many devices requiring a transparent contact for the foreseeable future. A full understanding of the origins of conductivity in these materials will prove essential to design and improve new and existing materials for these applications. Furthermore, such an understanding is required in order to optimzie the carrier density in these oxide materials for their use in the emergent field of transparent electronics. These considerations will also be vital in the control of conductivity in oxide nanostructures, which are expected to play an increasingly dominant role in future device applications.

\section{Acknowledgements}

We gratefully acknowledge numerous discussions on oxide semiconductors. In particular, we wish to thank M. W. Allen, F. Bechstedt, S. F. J. Cox, S. M. Durbin, R. G. Egdell, F. Fuchs, A. Janotti, S. Lany, R. L. Lichti, C. F. McConville, J. Robertson, D. O. Scanlon, A. Schleife, F. Tuomisto, C. G. Van de Walle, A. Walsh, G. W. Watson, and J. Zúñiga-Pérez. We are grateful to the Engineering and Physical Sciences Research Council, UK, for financial support. 
[1] Hogarth C A 1951 Nature 167 521-522

[2] Porch A, Morgan D V, Perks R M, Jones M O and Edwards P P 2004 J. Appl. Phys. 95 4734-4737

[3] Porch A, Morgan D V, Perks R M, Jones M O and Edwards P P 2004 J. App. Phys. 96 4211-4218

[4] Tiwari A N, Khrypunov G, Kurdzesau F, Btzner D L, Romeo A and Zogg H 2004 Progress in Photovoltaics: Research and Applications 12 33-38

[5] Chae G S 2001 Jpn. J. Appl. Phys. 40 1282-1286

[6] Hamberg I and Granqvist C G 1986 J. Appl. Phys. 60 R123-R160

[7] Granqvist C G and Hultker A 2002 Thin Solid Films 411 1-5

[8] Azens A and Granqvist C 2003 J. Solid State Electrochem. 7 64-68

[9] Ponce F A and Bour D P 1997 Nature 386 351-359

[10] Wu J, Walukiewicz W, Yu K M, Shan W, Ager III J W, Haller E E, Lu H, Schaff W J, Metzger W K and Kurtz S 2003 J. Appl. Phys. 94 6477-6482

[11] Schubert E F and Kim J K 2005 Science 308 1274-1278

[12] Wu J 2009 J. Appl. Phys. 106011101

[13] Service R F 2010 Science 327 1598-1599

[14] Minami T 2005 Semicond. Sci. Technol. 20 S35-S44

[15] Theis T N and Solomon P M 2010 Science 327 1600-1601

[16] Thomas G 1997 Nature 389 907-908

[17] Wager J F 2003 Science 300 1245-1246

[18] Ramirez A P 2007 Science 315 1377-1378

[19] Heber J 2009 Nature 459 28-30

[20] Takagi H and Hwang H Y 2010 Science 327 1601-1602

[21] Mannhart J and Schlom D G 2010 Science 327 1607-1611

[22] Ohta H and Hosono H 2004 Materials Today 7 42-51

[23] Nomura K, Ohta H, Ueda K, Kamiya T, Hirano M and Hosono H 2003 Science 300 1269-1272

[24] Jeong J K, Jeong J H, Yang H W, Park J S, Mo Y G and Kim H D 2007 Appl. Phys. Lett. 91 113505

[25] Kim G H, Ahn B D, Shin H S, Jeong W H, Kim H J and Kim H J 2009 Appl. Phys. Lett. 94 233501

[26] Tsukazaki A, Ohtomo A, Kita T, Ohno Y, Ohno H and Kawasaki M 2007 Science 315 1388-1391

[27] Tsukazaki A, Akasaka S, Nakahara K, Ohno Y, Ohno H, Maryenko D, Ohtomo A and Kawasaki M 2010 Nature Mater. 9 889-893 ISSN 1476-1122

[28] von Klitzing K 1986 Rev. Mod. Phys. 58 519-531

[29] von Klitzing K, Dorda G and Pepper M 1980 Phys. Rev. Lett. 45 494-497

[30] Schlom D G and Pfeiffer L N 2010 Nature Mater. 9 881-883 ISSN 1476-1122

[31] Ohtomo A and Hwang H Y 2004 Nature 427 423-426

[32] Brinkman A, Huijben M, van Zalk M, Huijben J, Zeitler U, Maan J C, van der Wiel W G, Rijnders G, Blank D H A and Hilgenkamp H 2007 Nature Mater. 6 493-496 ISSN 1476-1122

[33] Reyren N, Thiel S, Caviglia A D, Kourkoutis L F, Hammerl G, Richter C, Schneider C W, Kopp T, Ruetschi A S, Jaccard D, Gabay M, Muller D A, Triscone J M and Mannhart J 2007 Science 317 1196-1199

[34] Bourlange A, Payne D J, Egdell R G, Foord J S, Edwards P P, Jones M O, Schertel A, Dobson P J and Hutchison J L 2008 Appl. Phys. Lett. 92092117

[35] White M E, Tsai M Y, Wu F and Speck J S 2008 J. Vac. Sci. Technol. A 26 1300-1307

[36] Bourlange A, Payne D, Palgrave R, Foord J, Egdell R, Jacobs R, Schertel A, Hutchison J and Dobson P 2009 Thin Solid Films $\mathbf{5 1 7}$ 4286-4294

[37] Jalan B, Moetakef P and Stemmer S 2009 Appl. Phys. Lett. 95032906

[38] Jalan B, Engel-Herbert R, Wright N J and Stemmer S 2009 J. Vac. Sci. Technol. A 27 461-464

[39] Son J, Moetakef P, Jalan B, Bierwagen O, Wright N J, Engel-Herbert R and Stemmer S 2010 Nature Mater. 9 482-484

[40] Bierwagen O and Speck J S 2010 J. Appl. Phys. 107113519 
[41] Samson S and Fonstad C G 1973 J. Appl. Phys. 44 4618-4621

[42] Godby R W, Schlüter M and Sham L J 1986 Phys. Rev. Lett. 56 2415-2418

[43] Hine N D M, Frensch K, Foulkes W M C and Finnis M W 2009 Phys. Rev. B 79024112

[44] Lany S and Zunger A 2009 Modelling Simul. Mater. Sci. Eng. 17084002

[45] Lany S and Zunger A 2008 Phys. Rev. B 78235104

[46] McCluskey M D and Jokela S J 2009 J. Appl. Phys. 106071101

[47] Evans S M, Giles N C, Halliburton L E and Kappers L A 2008 J. Appl. Phys. 103043710

[48] Vlasenko L S and Watkins G D 2005 Phys. Rev. B 71125210

[49] Janotti A and de Walle C G V 2005 Appl. Phys. Lett. 87122102

[50] Tuomisto F, Saarinen K, Look D C and Farlow G C 2005 Phys. Rev. B 72085206

[51] Janotti A and Van de Walle C G 2007 Phys. Rev. B 76165202

[52] Lee W J, Kang J and Chang K J 2006 Phys. Rev. B 73024117

[53] Lany S and Zunger A 2007 Phys. Rev. Lett. 98045501

[54] Erhart P, Albe K and Klein A 2006 Phys. Rev. B 73205203

[55] Selim F A, Weber M H, Solodovnikov D and Lynn K G 2007 Phys. Rev. Lett. 99085502

[56] Kılıç Ç and Zunger A 2002 Phys. Rev. Lett. 88095501

[57] Singh A K, Janotti A, Scheffler M and de Walle C G V 2008 Phys. Rev. Lett. 101055502

[58] Limpijumnong S, Reunchan P, Janotti A and Van de Walle C G 2009 Phys. Rev. B 80193202

[59] Varley J B, Weber J R, Janotti A and de Walle C G V 2010 Appl. Phys. Lett. 97142106

[60] de Wit J H W 1975 J. Solid State Chem. 13 192-200

[61] de Wit J H W 1977 J. Solid State Chem. 20 143-148

[62] Mizusaki J, Koinuma H, Shimoyama J I, Kawasaki M and Fueki K 1990 J. Solid State Chem. 88 443-450

[63] King P D C, Veal T D, Jefferson P H, Zúñiga-Pérez J, Muñoz-Sanjosé V and McConville C F 2009 Phys. Rev. B $\mathbf{7 9} 035203$

[64] Speaks D T, Mayer M A, Yu K M, Mao S S, Haller E E and Walukiewicz W 2010 J. Appl. Phys. 107113706 (pages 5)

[65] Zubiaga A, Tuomisto F, Coleman V A, Tan H H, Jagadish C, Koike K, Sasa S, Inoue M and Yano M 2008 Phys. Rev. B $\mathbf{7 8} 035125$

[66] Kröger F A 1974 The Chemistry of Imperfect Crystals (Amsterdam: North-Holland)

[67] Tomlins G W, Routbort J L and Mason T O 2000 J. Appl. Phys. 87 117-123

[68] Godinho K G, Walsh A and Watson G W 2009 J. Phys. Chem. C 113 439-448

[69] Look D C, Hemsky J W and Sizelove J R 1999 Phys. Rev. Lett. 82 2552-2555

[70] Erhart P and Albe K 2006 Appl. Phys. Lett. 88201918

[71] Vlasenko L S and Watkins G D 2005 Phys. Rev. B 72035203

[72] Tuomisto F, Ranki V, Saarinen K and Look D C 2003 Phys. Rev. Lett. 91205502

[73] Kim Y S and Park C H 2009 Phys. Rev. Lett. 102086403

[74] Tang L M, Wang L L, Wang D, Liu J Z and Chen K Q 2010 J. Appl. Phys. 107083704

[75] Look D C, Farlow G C, Reunchan P, Limpijumnong S, Zhang S B and Nordlund K 2005 Phys. Rev. Lett. 95225502

[76] Zhang S B, Wei S H and Zunger A 2001 Phys. Rev. B 63075205

[77] Lany S and Zunger A 2005 Phys. Rev. B 72035215

[78] Pankove J I and Johnson N M 1991 Semiconductors and Semimetals Vol. 34, Hydrogen in Semiconductors (Boston: Academic)

[79] Van de Walle C G and Neugebauer J 2003 Nature 423 626-628

[80] Pavesi L and Giannozzi P 1992 Phys. Rev. B 46 4621-4629

[81] Thomas D G and Lander J J 1956 J. Chem. Phys. 25 1136-1142

[82] Van de Walle C G 2000 Phys. Rev. Lett. 85 1012-1015

[83] Janotti A and Van de Walle C G 2007 Nature Mater. 6 44-47

[84] Cox S F J, Davis E A, Cottrell S P, King P J C, Lord J S, Gil J M, Alberto H V, Vilão R C, Piroto Duarte J, Ayres de Campos N, Weidinger A, Lichti R L and Irvine S J C 2001 Phys. 
Rev. Lett. 86 2601-2604

[85] Cox S F J 2009 Rep. Prog. Phys. 72116501

[86] Kreitzman S R, Hitti B, Lichti R L, Estle T L and Chow K H 1995 Phys. Rev. B 51 13117-13137

[87] Lichti R L, Bani-Salameh H N, Carroll B R, Chow K H, Hitti B and Kreitzman S R 2007 Phys. Rev. B 76045221

[88] Hofmann D M, Hofstaetter A, Leiter F, Zhou H, Henecker F, Meyer B K, Orlinskii S B, Schmidt J and Baranov P G 2002 Phys. Rev. Lett. 88045504

[89] Shimomura K, Nishiyama K and Kadono R 2002 Phys. Rev. Lett. 89255505

[90] Lavrov E V, Weber J, Börrnert F, Van de Walle C G and Helbig R 2002 Phys. Rev. B 66165205

[91] Seager C H and Myers S M 2003 J. APpl. Phys. 94 2888-2894

[92] Jokela S J, McCluskey M D and Lynn K G 2003 Physica B 340-342 221-224

[93] Lavrov E V, Börrnert F and Weber J 2005 Phys. Rev. B $\mathbf{7 1} 035205$

[94] Lavrov E V, Börrnert F and Weber J 2005 Phys. Rev. B 72085212

[95] Jokela S J and McCluskey M D 2005 Phys. Rev. B 72113201

[96] Shi G A, Stavola M, Pearton S J, Thieme M, Lavrov E V and Weber J 2005 Phys. Rev. B 72 195211

[97] Lavrov E V, Herklotz F and Weber J 2009 Phys. Rev. B 79165210

[98] Lavrov E V, Herklotz F and Weber J 2009 Phys. Rev. Lett. 102185502

[99] Chen L-Y, Chen W-H, Wang J-J, Hong F C-N 2004 Appl. Phys. Lett. 855628

[100] Sagar P, Kumar M, and Mehra R M 2005 Thin Solid Films 48994

[101] Kim D-H, Lee S-H, Lee G-H, Kim H-B, Kim K H, Lee Y-G, and Yu T-H 2010 J. Appl. Phys. 108023520

[102] King P D C, Lichti R L, Celebi Y G, Gil J M, ao R C V, Alberto H V, Duarte J P, Payne D J, Egdell R G, McKenzie I, McConville C F, Cox S F J and Veal T D 2009 Phys. Rev. B 80 081201(R)

[103] Cox S F J, Lord J S, Cottrell S P, Gil J M, Alberto H V, Keren A, Prabhakaran D, Scheuermann R and Stoykov A 2006 J. Phys.: Condens. Matter 18 1061-1078

[104] King P D C, McKenzie I and Veal T D 2010 Appl. Phys. Lett. 96062110

[105] Dakhel A A 2008 Semicond. Sci. Technol. 23055017

[106] Hlaing Oo W M, Tabatabaei S, McCluskey M D, Varley J B, Janotti A and Van de Walle C G 2010 Phys. Rev. B 82193201

[107] Koida T, Fujiwara H and Kondo M 2007 Jpn. J. Appl. Phys. 46 L685-L687

[108] Koida T, Fujiwara H and Kondo M 2009 Solar Energy Materials and Solar Cells 93 851-854

[109] Kılıç Ç and Zunger A 2002 Appl. Phys. Lett. 81 73-75

[110] Xiong K, Robertson J and Clark S J 2007 J. Appl. Phys. 102083710

[111] Ip K, Overberg M E, Heo Y W, Norton D P, Pearton S J, Stutz C E, Luo B, Ren F, Look D C and Zavada J M 2003 Appl. Phys. Lett. 82 385-387

[112] Wardle M G, Goss J P and Briddon P R 2006 Phys. Rev. Lett. 96205504

[113] Limpijumnong S and Van de Walle C G 2001 Phys. Stat. Sol. B 228 303-307

[114] Davis E A, Cox S F J, Lichti R L and Van de Walle C G 2003 Appl. Phys. Lett. 82 592-594

[115] Wu J, Walukiewicz W, Li S X, Armitage R, Ho J C, Weber E R, Haller E E, Lu H, Schaff W J, Barcz A and Jakiela R 2004 Appl. Phys. Lett. 84 2805-2807

[116] Gallinat C S, Koblmüller G, Brown J S, Bernardis S, Speck J S, Chern G D, Readinger E D, Shen H and Wraback M 2006 Appl. Phys. Lett. 89032109

[117] Losurdo M, Giangregorio M M, Bruno G, Kim T H, Choi S, Brown A S, Pettinari G, Capizzi M and Polimeni A 2007 Appl. Phys. Lett. 91081917

[118] Pettinari G, Masia F, Capizzi M, Polimeni A, Losurdo M, Bruno G, Kim T H, Choi S, Brown A, Lebedev V, Cimalla V and Ambacher O 2008 Phys. Rev. B 77125207

[119] Janotti A and Van de Walle C G 2008 Appl. Phys. Lett. 92032104

[120] King P D C, Veal T D and McConville C F 2009 J. Phys.: Condens. Matter 21174201

[121] Gallinat C S, Koblmüller G and Speck J S 2009 Appl. Phys. Lett. 95022103 
[122] Darakchieva V, Lorenz K, Barradas N P, Alves E, Monemar B, Schubert M, Franco N, Hsiao C L, Chen L C, Schaff W J, Tu L W, Yamaguchi T and Nanishi Y 2010 Appl. Phys. Lett. 96 081907

[123] Tamm I E 1932 Physikal. Z. Sowjetunion 1733

[124] Shockley W 1939 Phys. Rev. 56 317-323

[125] Maue A W 1935 Z. Physik 94717

[126] Mönch W 2001 Semiconductor surfaces and interfaces (Berlin: Springer)

[127] Allen F G and Gobeli G W 1962 Phys. Rev. 127 150-158

[128] Mönch W, Koke P and Krueger S 1981 J. Vac. Sci. Technol. 19 313-318

[129] Svensson S P, Kanski J, Andersson T G and Nilsson P O 1984 J. Vac. Sci. Technol. B 2 235-239

[130] Spicer W E, Lindau I, Gregory P E, Garner C M, Pianetta P and Chye P W 1976 J. Vac. Sci. Technol. 13 780-785

[131] Klein A 2000 Appl. Phys. Lett. 77 2009-2011

[132] Gassenbauer Y, Schafranek R, Klein A, Zafeiratos S, Hävecker M, Knop-Gericke A and Schlögl R 2006 Phys. Rev. B 73245312

[133] Harvey S P, Mason T O, Gassenbauer Y, Schafranek R and Klein A 2006 J. Phys. D: Appl. Phys. 39 3959-3968

[134] King P D C, Veal T D, Payne D J, Bourlange A, Egdell R G and McConville C F 2008 Phys. Rev. Lett. 101116808

[135] King P D C, Veal T D, Fuchs F, Wang C Y, Payne D J, Bourlange A, Zhang H, Bell G R, Cimalla V, Ambacher O, Egdell R G, Bechstedt F and McConville C F 2009 Phys. Rev. B 79205211

[136] Zhang K H L, Payne D, Palgrave R G, Lazarov V K, Chen W, Wee A T S, McConville C F, King P D C, Veal T D, Panaccione G, Lacovig P and Egdell R G 2009 Chem. Mater. 21 4353-4355

[137] Heiland G and Kunstmann P 1969 Surf. Sci. 13 72-84

[138] Coppa B J, Fulton C C, Hartlieb P J, Davis R F, Rodriguez B J, Shields B J and Nemanich R J 2004 J. Appl. Phys. 95 5856-5864

[139] Allen M W, Swartz C H, Myers T H, Veal T D, McConville C F and Durbin S M 2010 Phys. Rev. B 81075211

[140] Schmidt O, Kiesel P, Van de Walle C G, Johnson N M, Nause J and Döhler G H 2005 Jpn. J. Appl. Phys. 44 7271-7274

[141] Frésart E D, Darville J and Gilles J M 1983 Surf. Sci. 126 518-522

[142] Nagata T, Bierwagen O, White M E, Tsai M Y and Speck J S 2010 J. Appl. Phys. 107033707

[143] Fleischer M and Meixner H 1992 Sensors and Actuators B 7 257-261

[144] Geistlinger H 1994 Sensors and Actuators B 18-19 125-131

[145] Lovejoy T C, Yitamben E N, Shamir N, Morales J, Villora E G, Shimamura K, Zheng S, Ohuchi F S and Olmstead M A 2009 Appl. Phys. Lett. 94081906

[146] Look D C 2008 J. Appl. Phys. 104063718

[147] Look D C, Claflin B and Smith H E 2008 Appl. Phys. Lett. 92122108

[148] Meevasana W, King P D C, He R H, Mo S K, Hashimoto M, Tamai A, Songsiriritthigul P, Baumberger F and Shen Z X 2011 Nature Materials 10114

[149] Santander-Syro A F, Copie O, Kondo T, Fortuna F, Pailhes S, Weht R, Qiu X G, Bertran F, Nicolaou A, Taleb-Ibrahimi A, Fevre P L, Herranz G, Bibes M, Apertet Y, Lecoeur P, Rozenberg M J and Barthelemy A 2011 Nature 469189

[150] King P D C, Veal T D, McConville C F, Zúñiga Pérez J, Muñoz Sanjosé V, Hopkinson M, Rienks E D L, Jensen M F and Hofmann P 2010 Phys. Rev. Lett. 104256803

[151] Piper L F J, Colakerol L, King P D C, Schleife A, Zúñiga-Pérez J, Glans P A, Learmonth T, Federov A, Veal T D, Fuchs F, Muñoz-Sanjosé V, Bechstedt F, McConville C F and Smith K E 2008 Phys. Rev. B 78165127

[152] Piper L F J, Veal T D, McConville C F, Lu H and Schaff W J 2006 Appl. Phys. Lett. 88252109

[153] Cimalla V, Lebedev V, Morales F M, Goldhahn R and Ambacher O 2006 Appl. Phys. Lett. 89 172109 
[154] Lebedev V, Cimalla V, Baumann T, Ambacher O, Morales F M, Lozano J G and González D 2006 J. Appl. Phys. 100094903

[155] Zhang K H L, Lazarov V K, Veal T D, Oropeza F E, McConville C F, Egdell R G and Walsh A 2011 J. Phys: Condens. Matter. this issue

[156] Tersoff J 1984 Phys. Rev. Lett. 52 465-468

[157] Tejedor C and Flores F 1977 J. Phys. C 11 L19

[158] Heine V 1965 Phys. Rev. 138 A1689-A1696

[159] Inkson J C 1980 J. Phys. C 13 369-381

[160] Walukiewicz W 1988 J. Vac. Sci. Technol. B 6 1257-1262

[161] Walukiewicz W 1989 Appl. Phys. Lett. 54 2094-2096

[162] Walukiewicz W 1988 Phys. Rev. B 37 4760-4763

[163] Veal T D, King P D C, Hatfield S A, Bailey L R, McConville C F, Martel B, Moreno J C, Frayssinet E, Semond F and Zúñiga-Pérez J 2008 Appl. Phys. Lett. 93202108

[164] King P D C, Veal T D, Jefferson P H, McConville C F, Wang T, Parbrook P J, Lu H and Schaff W J 2007 Appl. Phys. Lett. 90132105

[165] King P D C, Veal T D, Kendrick C E, Bailey L R, Durbin S M and McConville C F 2008 Phys. Rev. B 78033308

[166] Falabretti B and Robertson J 2007 J. Appl. Phys. 102123703

[167] Schleife A, Fuchs F, Rödl C, Furthmüller J and Bechstedt F 2009 Appl. Phys. Lett. 94012104

[168] Brudnyi V N, Grinyaev S N and Kolin N G 2003 Semiconductors 37 537-545

[169] King P D C, Veal T D, Jefferson P H, Hatfield S A, Piper L F J, McConville C F, Fuchs F, Furthmüller J, Bechstedt F, Lu H and Schaff W J 2008 Phys. Rev. B 77045316

[170] Fuchs F and Bechstedt F 2008 Phys. Rev. B 77155107

[171] Robertson J 1979 J. Phys. C: Solid State Phys. 124767

[172] Schleife A, Fuchs F, Furthmuller J and Bechstedt F 2006 Phys. Rev. B 73245212

[173] He H, Orlando R, Blanco M A, Pandey R, Amzallag E, Baraille I and Rérat M 2006 Phys. Rev. $B \mathbf{7 4} 195123$ (pages 8)

[174] O'Neil D H, Walsh A, Jacobs R M J, Kuznetsov V L, Egdell R G and Edwards P P 2010 Phys. Rev. B $\mathbf{8 1} 085110$

[175] Tersoff J 1985 Phys. Rev. B 32 6968-6971

[176] Veal T D, Lowe M J and McConville C F 2002 Surf. Sci. 499 251-260

[177] Peles A, Janotti A and de Walle C G V 2008 Phys. Rev. B 78035204

[178] Kawazoe H, Yasukawa M, Hyodo H, Kurita M, Yanagi H and Hosono H 1997 Nature 389 939-942

[179] Medvedeva J E and Freeman A J 2005 Europhys. Lett. 69 583-587

[180] Medvedeva J E and Hettiarachchi C L 2010 arXiv:1002.4827

[181] Moss T S 1954 Proc. Phys. Soc. B 67775

[182] Burstein E 1954 Phys. Rev. 93 632-633

[183] Berggren K F and Sernelius B E 1981 Phys. Rev. B 24 1971-1986

[184] Hamberg I, Granqvist C G, Berggren K F, Sernelius B E and Engström L 1984 Phys. Rev. B 30 3240-3249

[185] Dou Y, Fishlock T, Egdell R G, Law D S L and Beamson G 1997 Phys. Rev. B 55 R13381-R13384

[186] Dou Y, Egdell R G, Walker T, Law D S L and Beamson G 1998 Surf. Sci. 398 241-258

[187] Egdell R G, Walker T J and Beamson G 2003 J. Electron Spectrosc. Relat. Phenom. 128 59-66

[188] Lu J G, Fujita S, Kawaharamura T, Nishinaka H, Kamada Y, Ohshima T, Ye Z Z, Zeng Y J, Zhang Y Z, Zhu L P, He H P and Zhao B H 2007 J. Appl. Phys. 101083705

[189] Jefferson P H, Hatfield S A, Veal T D, King P D C, McConville C F, Zúñiga-Pérez J and MuñozSanjosé V 2008 Appl. Phys. Lett. 92022101

[190] Walsh A, Silva J L F D and Wei S H 2008 Phys. Rev. B 78075211

[191] Jaffe J E, Pandey R and Kunz A B 1991 Phys. Rev. B 43 14030-14034

[192] Walsh A, Silva J L F D, Wei S H, Körber C, Klein A, Piper L F J, DeMasi A, Smith K E, Panaccione G, Torelli P, Payne D J, Bourlange A and Egdell R G 2008 Phys. Rev. Lett. 100 
167402

[193] Fröhlich D, Kenklies R and Helbig R 1978 Phys. Rev. Lett. 41 1750-1751

[194] Segev D and Wei S H 2005 Phys. Rev. B 71125129

[195] Walsh A, Silva J L F D, Yan Y, Al-Jassim M M and Wei S H 2009 Phys. Rev. B 79073105

[196] Walsh A, Da Silva J L F and Wei S H 2011 J. Phys: Condens. Matter. this issue

[197] Heeger A J 2001 Rev. Mod. Phys. 73 681-700

[198] MacDiarmid A G 2001 Rev. Mod. Phys. 73 701-712

[199] Shirakawa H 2001 Rev. Mod. Phys. 73 713-718

[200] Groenendaal L, Jonas F, Freitag D, Pielartzik H and Reynolds J R 2000 Adv. Mater. 12 481-494

[201] Wu Z, Chen Z, Du X, Logan J M, Sippel J, Nikolou M, Kamaras K, Reynolds J R, Tanner D B, Hebard A F and Rinzler A G 2004 Science 305 1273-1276

[202] Zhang M, Fang S, Zakhidov A A, Lee S B, Aliev A E, Williams C D, Atkinson K R and Baughman R H 2005 Science 309 1215-1219 

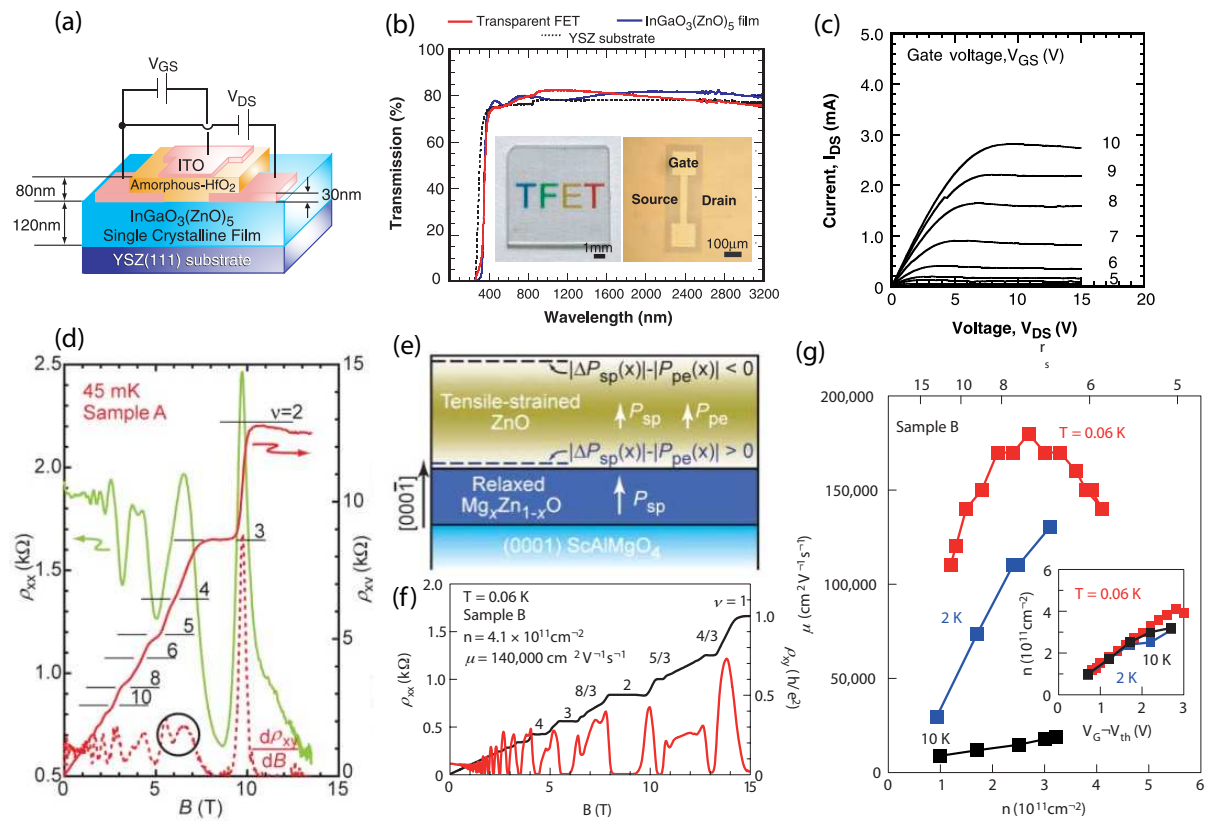

Figure 1. (a) Schematic device structure, (b) optical transmission spectrum, and (c) output characteristics of an $\mathrm{InGaO}_{3}(\mathrm{ZnO})_{5}$-based transparent field-effect transistor. (d) Longitudinal resistivity, $\rho_{x x}$, Hall resistivity, $\rho_{x y}$, and differential Hall resistivity $\mathrm{d} \rho_{x y} / \mathrm{d} B$ vs. field $B$ showing the quantum Hall effect measured at $45 \mathrm{mK}$ in $\mathrm{ZnO} / \mathrm{MgZnO}$ heterostructures, with the schematic device structure shown in (e). (f) $\rho_{x x}$ and $\rho_{x y}$ vs $B$ measured at $60 \mathrm{mK}$ showing the fractional quantum Hall effect in purer heterostructures, with mobility as shown in (g). [(a-c): From K. Nomura, H. Ohta, K. Ueda, T. Kamiya, M. Hirano, and H. Hosono, Science 300, 1269 (2003). Reprinted with permission from AAAS. (d) and (e): From A. Tsukazaki, A. Ohtomo, T. Kita, Y. Ohno, H. Ohno, and M. Kawasaki, Science 315, 1388 (2007). Reprinted with permission from AAAS. (f) and (g): Reprinted by permission from Macmillan Publishers Ltd: A. Tsukazaki, S. Akasaka, K. Nakahara, Y. Ohno, H. Ohno, D. Maryenko, A. Ohtomo, and M. Kawasaki, Nature Materials 9, 889 (2010), copyright 2010.] 


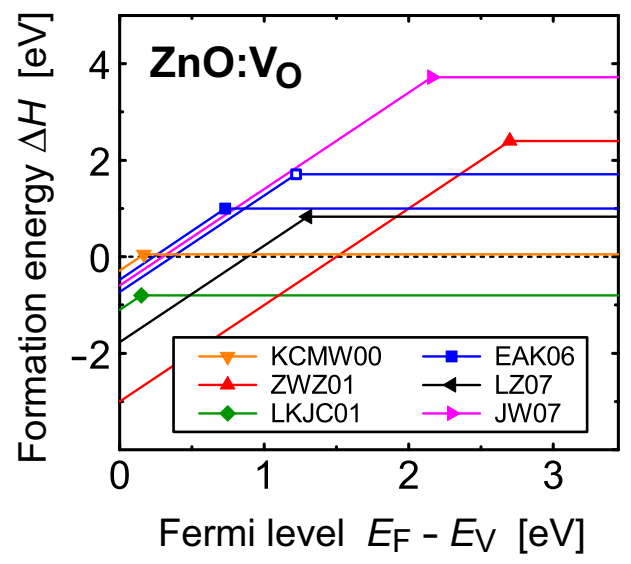

Figure 2. Formation energies for the oxygen vacancy in $\mathrm{ZnO}$ calculated using different schemes to account for supercell finite-size effects and band gap deficiencies within DFT. For an explanation of the symbols, see Fig. 1 of Ref. [45]. Reprinted with permission from S. Lany and A. Zunger, Physical Review B, 78, 235104 (2008). Copyright 2008 by the American Physical Society.

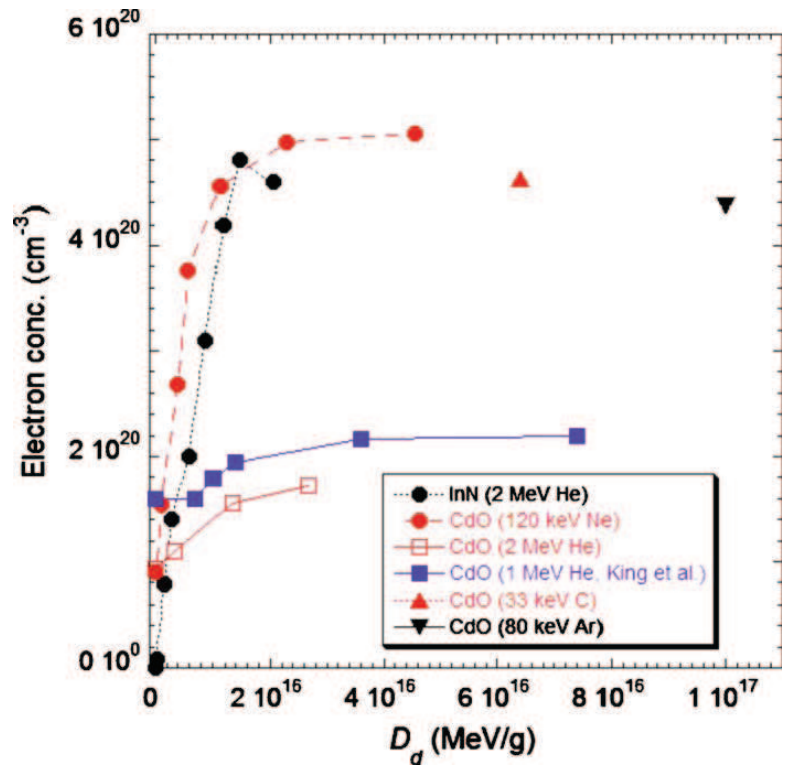

Figure 3. Evolution of carrier concentration in $\mathrm{CdO}$ when irradiated with highenergy ions, in order to introduce native defects. Reprinted with permission from D. T. Speaks, M. A. Mayer, K. M. Yu, S. S. Mao, E. E. Haller, and W. Walukiewicz, Journal of Applied Physics, 107, 113706 (2010). Copyright 2010, American Institute of Physics. 
(a)

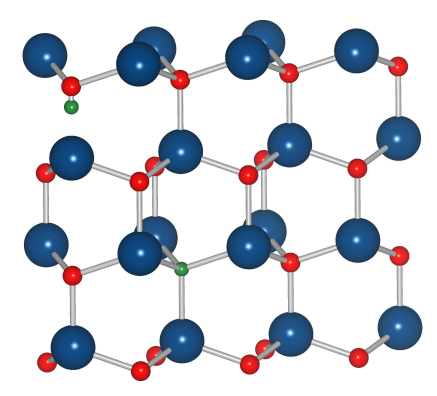

(b)

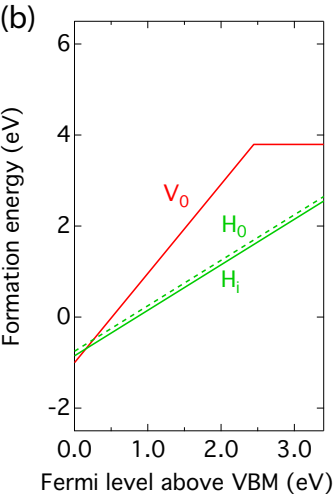

Figure 4. (a) Schematic representation of interstitial and substitutional hydrogen impurities in $\mathrm{ZnO}$, and (b) corresponding calculated formation energies [83] compared to that of the oxygen vacancy.

(a)
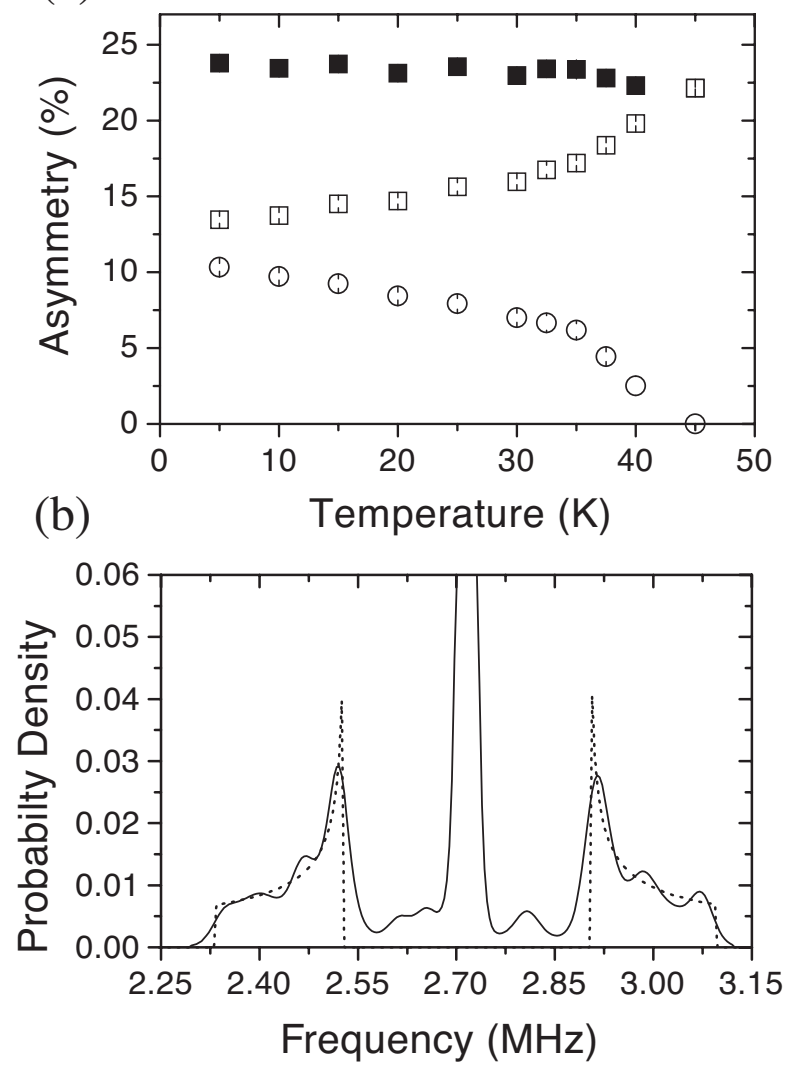

Figure 5. (a) Observation of paramagnetic muonium, $\mathrm{Mu}^{0}$ (open circles), diamagnetic muonium, $\mathrm{Mu}^{+}$(open squares), and the total muonium amplitude (solid squares) in $\mathrm{ZnO}$ derived from transverse-field $\mu \mathrm{SR}$ measurements. (b) A frequency-domain maximum entropy transform of the time-domain muon spin rotation signal measured at $5 \mathrm{~K}$, showing satellites to the central diamagnetic line due to the paramagnetic component. The dashed line is the expected frequency distribution. Reprinted with permission from S. F. J. Cox, E. A. Davis, S. P. Cottrell, P. J. C. King, J. S. Lord, J. M. Gil, H. V. Alberto, R. C. Vilão, J. Piroto Duarte, N. Ayres de Campos, A. Weidinger, R. L. Lichti, and S. J. C. Irvine, Physical Review Letters, 86, 2601 (2001). Copyright 2001 by the American Physical Society. 


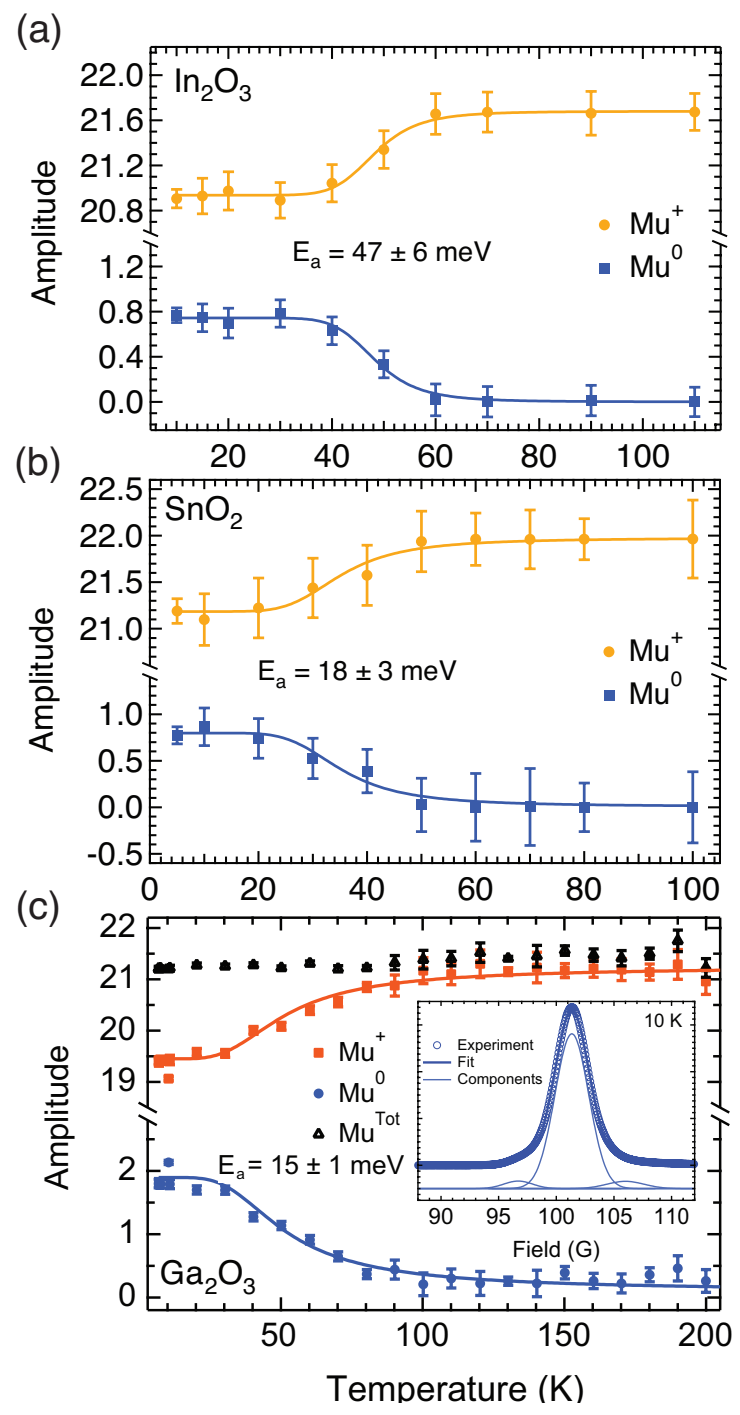

Figure 6. (a) Observation of paramagnetic muonium, $\mathrm{Mu}^{0}$, and diamagnetic muonium, $\mathrm{Mu}^{+}$, in (a) $\mathrm{In}_{2} \mathrm{O}_{3}$, (b) $\mathrm{SnO}_{2}$, and (c) $\mathrm{Ga}_{2} \mathrm{O}_{3}$, derived from transversefield $\mu \mathrm{SR}$ measurements. A frequency-domain maximum entropy transform of the $\mathrm{Ga}_{2} \mathrm{O}_{3}$ time-domain muon spin rotation signal measured at $10 \mathrm{~K}$, showing small closely spaced paramagnetic satellites to the central diamagnetic line, is shown inset to (c). $(a, b)$ : Reprinted with permission from P. D. C. King, R. L. Lichti, Y. G. Celebi, J. M. Gil, R. C. Vilão, H. V. Alberto, J. P. Duarte, D. J. Payne, R. G. Egdell, I. McKenzie, C. F. McConville, S. F. J. Cox, and T. D. Veal, Physical Review B, 80, 081201(R) (2009). Copyright 2009 by the American Physical Society. (c): Reprinted with permission from P. D. C. King, I. McKenzie, and T. D. Veal, Applied Physics Letters, 96062110 (2010). Copyright 2010, American Institute of Physics. 


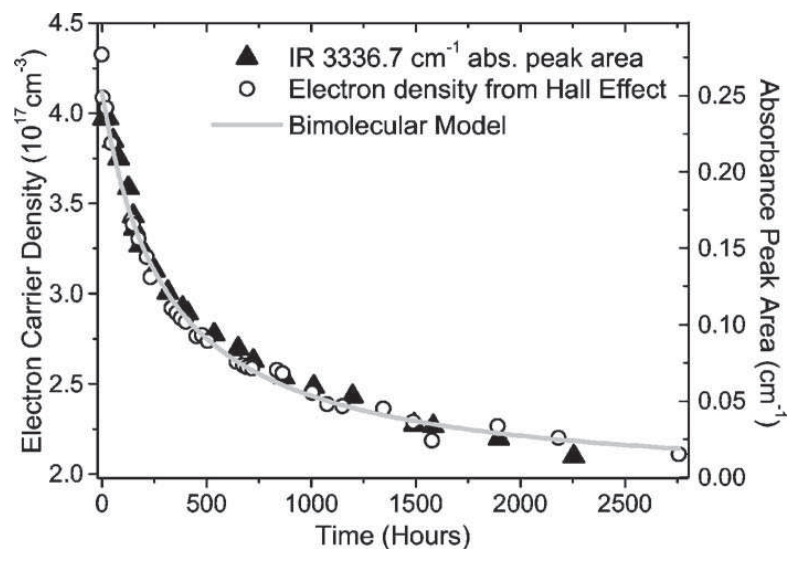

Figure 7. Room temperature evolution of the O-H IR absorbance peak area and carrier concentration in hydrogenated $\mathrm{ZnO}$ with time. A bimolecular model is also shown. Reprinted with permission from S. J. Jokela and M. D. McCluskey, Physical Review B, 72, 113201 (2005). Copyright 2005 by the American Physical Society.
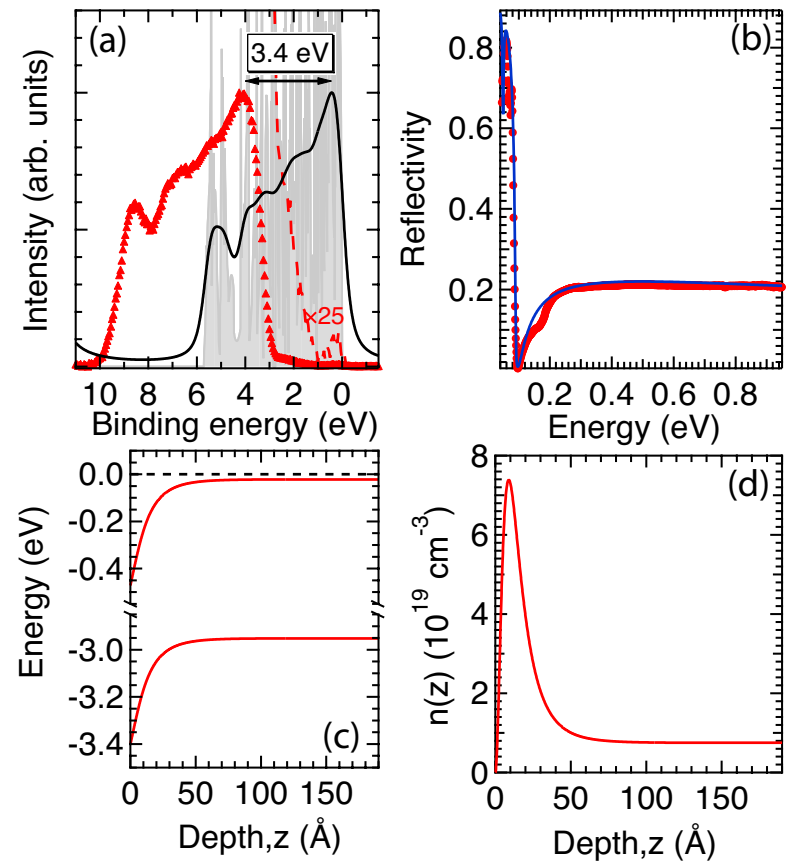

Figure 8. Surface electron accumulation in $\operatorname{In}_{2} \mathrm{O}_{3}$ : (a) valence-band photoemission, compared to density-functional theory calculations, revealing the Fermi level to be pinned $3.4 \mathrm{eV}$ above the VBM at the surface; (b) measured (circles) and simulated (line) IR-reflectivity, revealing a bulk Fermi level very close to the CBM, in agreement with Hall effect measurements; (c) downward band bending and (d) increase in carrier concentration in the resulting surface electron accumulation layer. Figure adapted from Refs. [134,135]. 


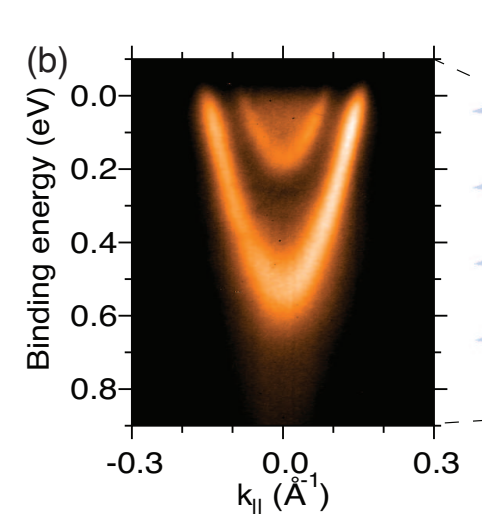

(a)
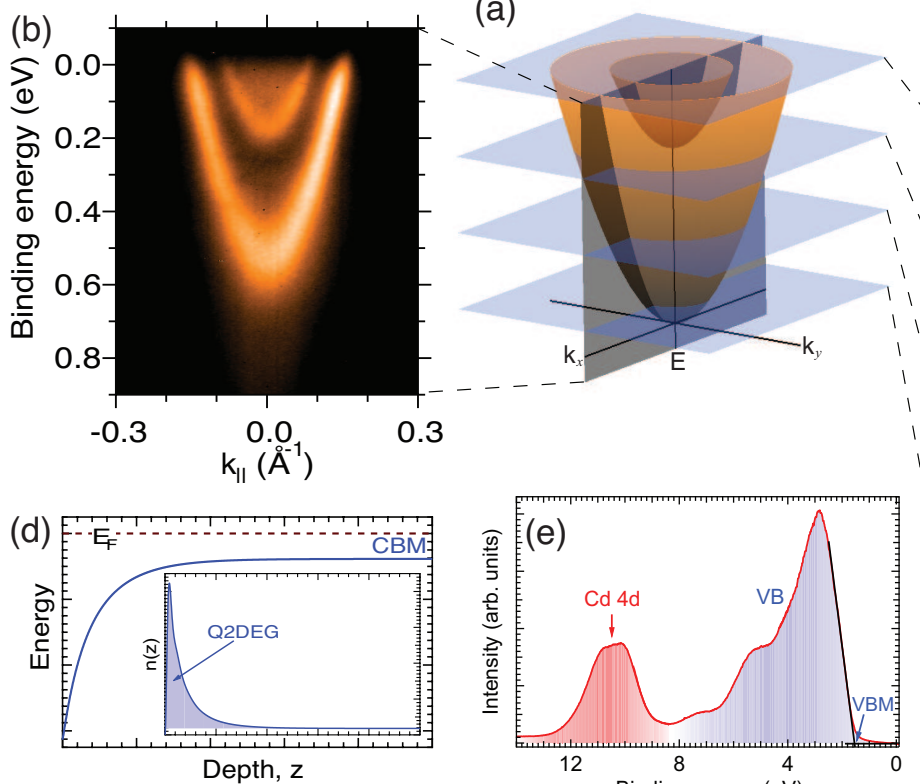

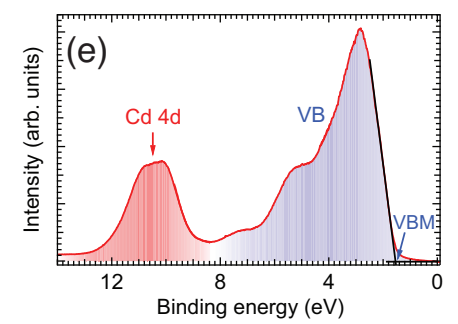

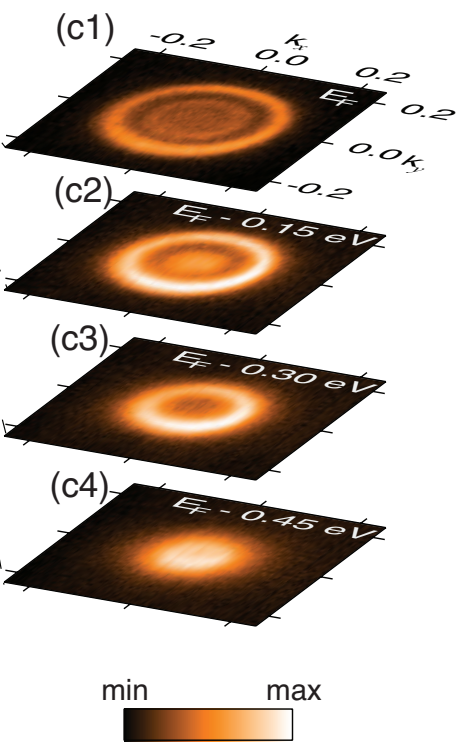

Figure 9. Quantized nature of electron accumulation at a CdO surface. (a) Schematic of the quantized subbands of a surface 2DEG (electron accumulation layer). (b) Dispersion and (c) constant energy contours of 2DEG states at the $\mathrm{CdO}(001)$ surface measured by ARPES. (d) Schematic representation of the downward band bending generic to TCO surfaces giving rise to the 2DEG (inset). (e) Angle-integrated valence band and core-level photoemission. Reprinted with permission from P. D. C. King, T. D. Veal, C. F. McConville, J. Zúñiga-Pérez, V. Muñoz-Sanjosé, M. Hopkinson, E. D. L. Rienks, M. F. Jensen, and Ph. Hofmann, Physical Review Letters, 104, 256803 (2010). Copyright 2010 by the American Physical Society. 


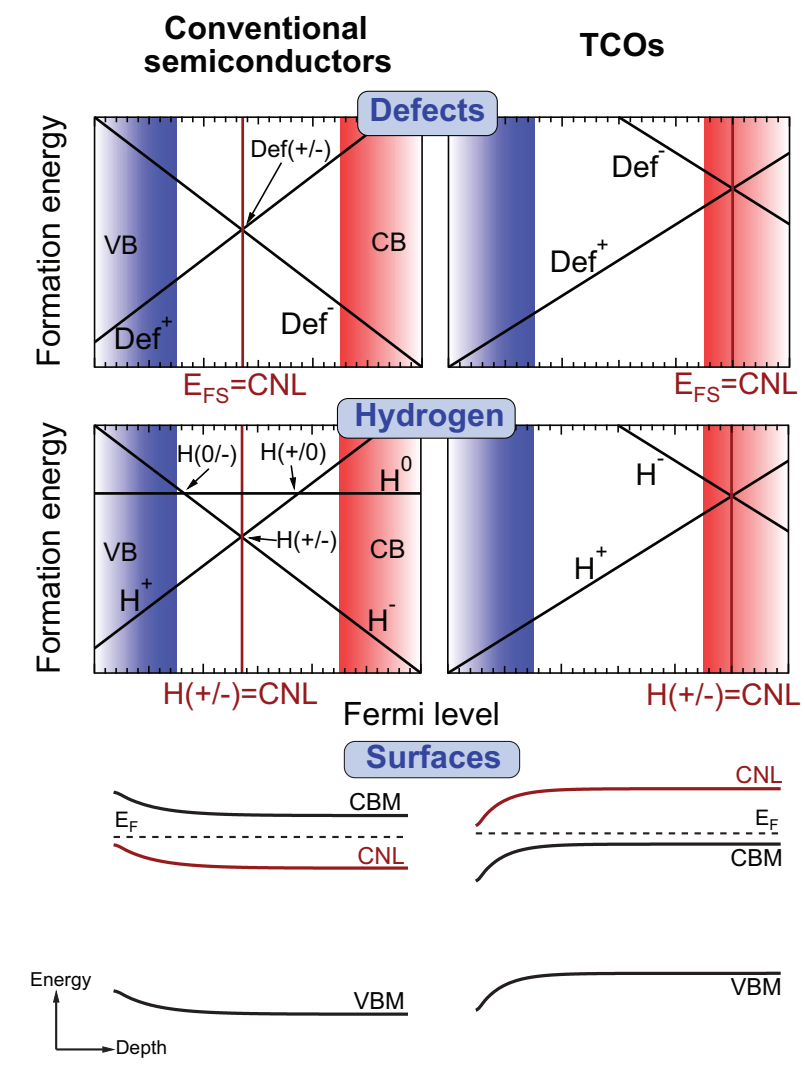

Figure 10. Schematic formation energies for dominant native defects and interstitial hydrogen impurities and schematic surface band bending in conventional semiconductors (left) and TCOs (right). The influence of the CNL, within the band gap in conventional semiconductors but above the CBM in TCOs, can be seen. Figure adapted from Ref. [63].

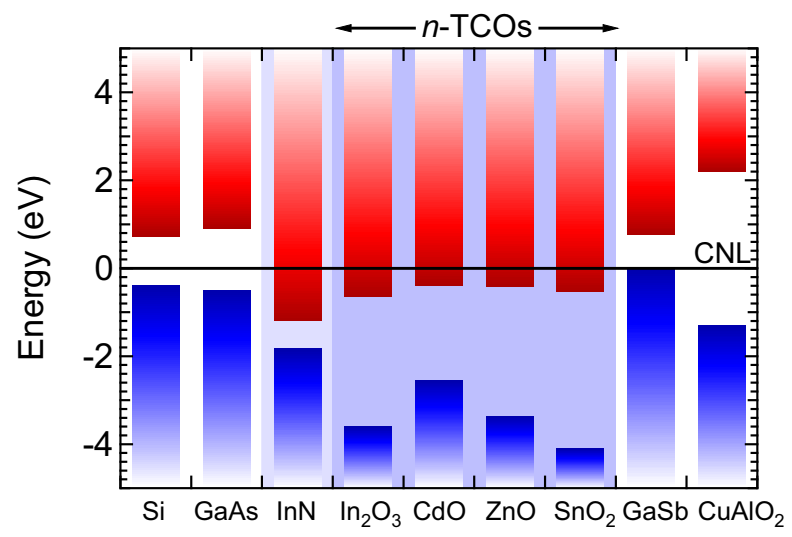

Figure 11. Band lineup of a number of TCOs and other semiconductors. The positions of the bands relative to the CNL are derived from the measurements and calculations in Refs. [63, 135, 163-166,168,169]. 


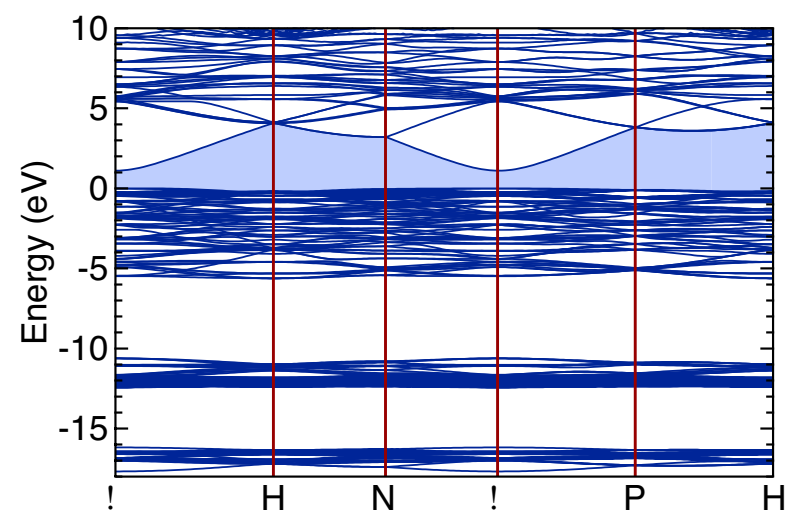

Figure 12. Calculated band structure of $\operatorname{In}_{2} \mathrm{O}_{3}$. Figure adapted from Ref. [170].

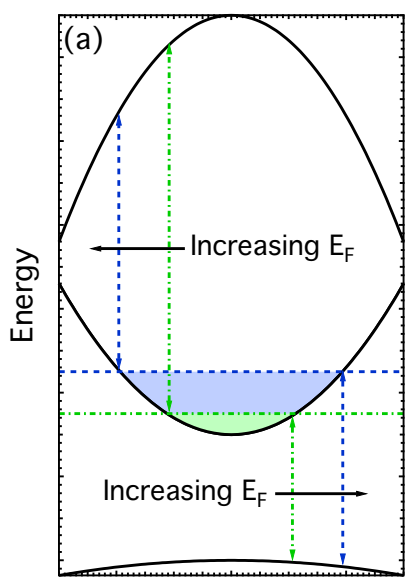

Wavevector

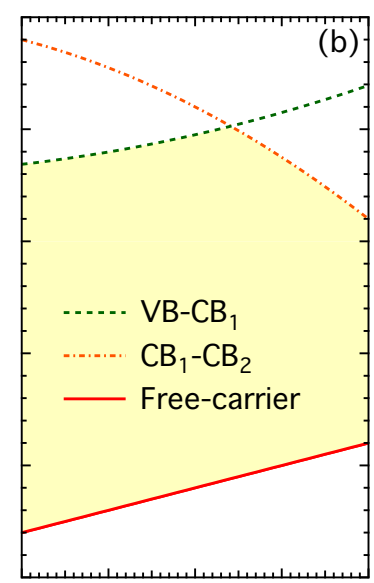

Carrier concentration

Figure 13. (a) Schematic of interband optical absorption, and its evolution with doping. (b) Shift of fundamental interband and free-carrier absorption edges as a function of free carrier concentration. The transparent region is shaded. 
(a)

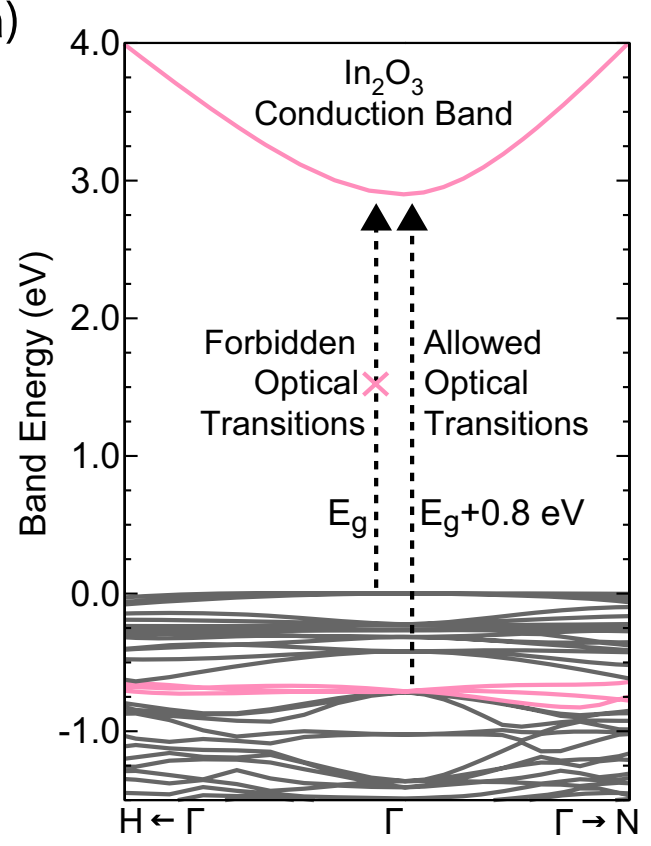

(b)

k-point

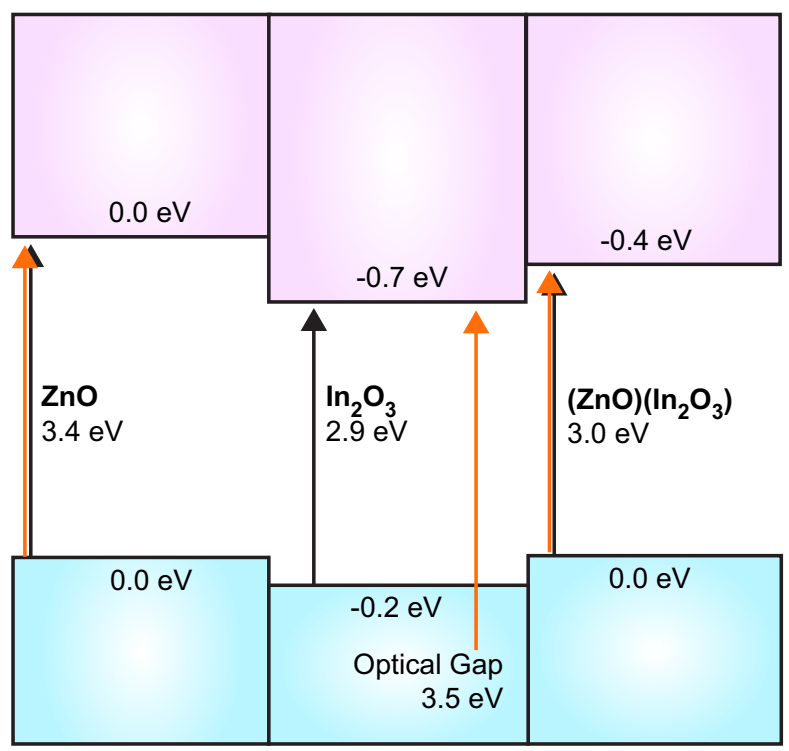

Figure 14. (a) Calculated band structure around the zone centre of $\mathrm{In}_{2} \mathrm{O}_{3}$ indicating the allowed and forbidden dipole transitions, adapted from Ref. [192], and (b) schematic of the band offsets and changes in allowed transitions with alloying in $\mathrm{ZnO}$, $\mathrm{In}_{2} \mathrm{O}_{3}$, and $\mathrm{In}_{2} \mathrm{O}_{3}(\mathrm{ZnO})_{n}$, adapted from Ref. [195]. 